\title{
Who Increases Emergency Department Use? New Insights from the Oregon Health Insurance Experiment*
}

\author{
Augustine Denteh $^{\dagger} \quad$ Helge Liebert ${ }^{\ddagger}$
}

April 11, 2023

\begin{abstract}
We provide new insights regarding the headline result that Medicaid increased emergency department (ED) use from the Oregon experiment. We find meaningful heterogeneous impacts of Medicaid on ED use using causal machine learning methods. The individualized treatment effect distribution includes a wide range of negative and positive values, suggesting the average effect masks substantial heterogeneity. A small group — about 14\% of participants-in the right tail of the distribution drives the overall effect. We identify priority groups with economically significant increases in ED usage based on demographics and previous utilization. Intensive margin effects are an important driver of increases in ED utilization.
\end{abstract}

Keywords: Medicaid, ED use, effect heterogeneity, causal machine learning, optimal policy JEL No: H75, I13, I38

\footnotetext{
*We thank Amy Finkelstein, Anirban Basu, Paul Goldsmith-Pinkham, Tymon Słoczyński, Timothy Layton, Jacob Wallace, Sherri Rose, Jason Fletcher, Carlos Lamarche, Mary Olson, Makayla Lavender, Prithvijit Mukherjee, Samuel Asare, Nicholas A. Wright, Anthony Strittmatter, Michael C. Knaus, and seminar/conference participants at Tulane University, University of Washington, University of Kentucky, Institute for Research on Poverty (University of Wisconsin-Madison), Swiss Health Economics Workshop, and The American Society of Health Economists (ASHEcon) for helpful comments.

${ }^{\dagger}$ Correspondence to Department of Economics, Tulane University, 6823 St. Charles Avenue, New Orleans, LA 70118; adenteh@tulane.edu.

ॠDepartment of Economics, University of Zurich, Schönberggasse 1, 8001 Zürich, Switzerland; helge.liebert@econ.uzh.ch.
} 


\section{Introduction}

The finding that Medicaid increased emergency department (ED) utilization in the 2008 Oregon Health Insurance Experiment drew widespread national attention (Taubman et al. 2014). Economic theory predicts that health insurance coverage reduces the out-of-pocket cost of care, leading to increased ED use. However, Medicaid may reduce ED use if people substitute primary care services for ED visits (Sommers \& Simon 2017). Empirically, the positive effect of Medicaid on ED use in Oregon contradicts most quasi-experimental studies that find a reduction in ED utilization following health insurance expansions (Chen et al. 2011, Miller 2012, Sommers et al. 2016, Chou et al. 2020, Giannouchos et al. 2022). ${ }^{1}$ Recently, Kowalski (2021) attempts to reconcile the contradictory findings in the Oregon experiment and the Massachusetts reform. Nonetheless, the effect of Medicaid on ED use, especially for non-urgent conditions, remains a crucial consideration in health insurance expansions because of the continued rise in ED visits, the declining number of emergency departments, and the effects of ED crowding on health (Tang et al. 2010, Moore \& Liang 2020, Woodworth 2020, Sabbatini \& Dugan 2022).

This paper provides new insights into how Medicaid affects ED utilization when we go beyond the average effect. Using the Oregon experiment, we estimate the heterogeneous impacts of Medicaid on ED use and characterize those driving the positive average effects. In 2008, Oregon randomly assigned the opportunity to apply for spots in its Oregon Health Plan Standard (OHP Standard). Unlike OHP Plus, which serves Oregon's typical Medicaid population, OHP Standard was newly offered to a group of uninsured adults who were categorically ineligible for Medicaid under federal guidelines (Allen et al. 2013). The Medicaid expansion increased utilization for most types of ED visits, including those not requiring immediate care, for up to two years (Taubman et al. 2014, Finkelstein et al. 2016). We do not revisit the average impacts of Medicaid that are well-documented in this literature. Instead, we extend the literature by investigating how Medicaid's effect on ED use varies by covariates, which helps to isolate the risk factors that matter for increased

${ }^{1}$ Garthwaite et al. (2019) is a notable exception that finds that the Affordable Care Act's Medicaid expansion increased ED use for "deferrable" conditions. 
utilization.

This paper makes three salient contributions. First, we nonparametrically estimate the heterogeneous treatment effects of Medicaid on ED utilization using causal machine learning methods. We estimate various types of heterogeneous effects, including individualized treatment effects that condition on individual covariate values and higher-level group average treatment effects using generalized random forests (Athey et al. 2019). Previous studies report a small number of subgroup effects for a subset of the Oregon experiment who completed a follow-up survey (Taubman et al. 2014). Subgroup analysis can miss interesting patterns of heterogeneity due to non-linear interactions between multiple covariates (Heckman et al. 1997, Angrist 2004, Deaton 2010). In addition to the need to pre-specify the covariates, subsample heterogeneity analysis is prone to multiple hypothesis testing problems (e.g. List et al. 2019, Young 2019). Considering that Oregon expanded Medicaid to a population not typically covered by it, insights from examining its heterogeneous effects can help interpret its findings and inform policy-making.

Second, we document the risk factors for increasing ED use upon Medicaid coverage, opening the doorway for identifying priority groups for targeted policy interventions. Unlike Kowalski (2021), we do not attempt to reconcile Oregon's ED finding with the Massachusetts health insurance reform results. In particular, Kowalski attributes the discrepancy to different local average treatment effects (LATE) arising from the same marginal treatment effect (MTE) function. In doing so, Kowalski estimates an MTE function for Oregon, assuming that treatment effects vary linearly with the fraction treated. The MTE function shows that treatment effects are positive for compliers but negative for never-takers. Kowalski (2021) then extrapolates the estimated MTE function to Massachusetts, finding that ED utilization is predicted to decrease for Massachusetts compliers comparable to a subset of Oregon never-takers. However, a related study, Marx (2020), shows that a different set of distribution assumptions within the same MTE framework predicts a positive treatment effect for Oregon's never-takers. Nonetheless, a key contribution of Kowalski (2021) is showing that treatment effect heterogeneity is crucial for understanding Medicaid's impact on ED use and transporting those results to new environments. Given the importance of heterogeneity, our paper complements the 
above studies by directly linking effect heterogeneity in Oregon to several observed characteristics in a data-driven manner without making additional assumptions beyond those needed to recover the LATE. Doing so sheds light on the drivers of Medicaid's positive average impact on ED use, providing new insights into the complex relationship between insurance coverage and ED use.

Third, we discuss the potential to leverage the heterogeneous effects of Medicaid to propose simple decision rules to identify enrollees for targeted educational/outreach interventions. Reducing "unnecessary" ED use (i.e., ED use for non-emergent conditions) is a high priority policy consideration for Medicaid policymakers. This is partly because one-third of ED visits are preventable and it costs up to five times less to treat the same health problems in a doctor's office (Adams 2013, Giannouchos et al. 2022). As such, policymakers are experimenting with various policies to curb unnecessary ED use among Medicaid recipients. For instance, as of 2020, about 14 states use blunt tools such as higher copayments to dissuade non-emergency ED use. ${ }^{2}$ Evidence of Medicaid's heterogeneous effects is essential for exploring targeted policy options (e.g., outreach programs) for reducing non-emergency ED use. However, policymakers might need guidance on how to use such evidence. Therefore, we illustrate one way of using heterogeneous treatment effects to estimate decision rules based on recently developed policy learning algorithms (Athey \& Wager 2021, Kitagawa \& Tetenov 2018). Such decision trees that optimize non-emergent ED use may be a promising way to identify enrollees at risk of greater non-urgent ED use for targeted outreach efforts.

Several broad findings emerge from our study. First, we find substantial heterogeneity in the impact of Medicaid coverage on ED visits. Individualized treatment effects span a wide range of negative and positive changes in ED utilization. Moreover, the statistically significant increases in overall ED use are concentrated in the right tail of the distribution. Specifically, for ED use on the extensive margin, a small group of people-about $14 \%$ of recipients—with statistically significant increases in ED visits appear to drive the positive average impact of Medicaid. We find similar patterns of heterogeneity for ED use on the intensive margin. These findings persist for various types of ED visits. In some cases, we find that average null effects hide important countervailing forces-

${ }^{2}$ See list of states at https://www.kff.org. 
reductions in ED use by some people offset increases in utilization by others. For instance, Taubman et al. (2014) find no effects on average for conditions classified as "emergent, not preventable" (i.e., illnesses that require immediate medical care and could not have been prevented). In this case, the individualized treatment effects show a large share of both statistically significant increases and decreases in ED use, culminating in the average null effect.

Second, the heterogeneous effects of Medicaid yield insights into who drives the average impact when we aggregate the individualized effects to higher group levels. The main risk factors for increased ED use are gender, age, participation in other safety net programs, and past ED use for conditions not requiring emergency care. Based on those factors, we identify four subgroups estimated to have statistically significant increases in ED use of at least twice the magnitude of the average effect. These groups are men, prior SNAP participants, younger adults under 50 years, and those with pre-lottery ED use classified as primary care treatable. We do not find statistically significant group-level effects defined by other pre-lottery ED use variables or information collected during the lottery sign-up. Overall, the results suggest that the increases in ED use occur primarily for newly insured people with a history of using the emergency department for conditions that do not require it and could be treated in primary care settings.

Finally, we illustrate one way to use the heterogeneous effects to estimate decision rules identifying those predicted to increase non-emergent ED use. Based on a small set of covariates, the policy learning algorithm identifies two groups of people at risk of increased ED use. The first group consists of people with minimal prior outpatient ED use but who did so for non-emergent conditions. The second group is those with higher previous outpatient ED visits who rarely use the ED for conditions requiring inpatient care. Policymakers can use those assignment rules to prioritize outreach to new recipients at risk of unnecessary ED use. While our decision rule reasonably identifies those unlikely to use ED care for emergencies, we caution that this exercise is illustrative. Policymakers must tailor policy-relevant decision rules to meet their objectives subject to legal and other practical constraints.

The rest of the paper is organized as follows. Section 2 describes the context of Oregon's health 
insurance experiment and the data. Section 3 presents the causal machine learning framework. Section 4 presents and discusses the results. Section 5 concludes. Additional results are collected in Appendices A and B.

\section{The Oregon Health Insurance Experiment}

\subsection{Background}

We provide a brief overview of the essential features of the Oregon Health Insurance Experiment. Previous studies contain detailed institutional information (Finkelstein et al. 2012, Baicker et al. 2013, Allen et al. 2013, Taubman et al. 2014, Finkelstein et al. 2016). Oregon split its Medicaid program into two due to a budget shortfall in 2003. The first program (OHP Plus) served categorically eligible people under federal rules-low-income income children, pregnant women, blind or disabled people, and Temporary Assistance to Needy Families (TANF) recipients. The second program (OHP Standard) served low-income and non-disabled adults not categorically eligible for the OHP Plus program. Oregon's philosophy was to provide more people with coverage for fewer services rather than limiting participation. Thus, OHP Standard covered fewer services and came with higher premium and cost-sharing requirements, resulting in a decline in enrolment (Allen et al. 2013). The resulting attrition in OHP Standard participation led to an accumulated budgetary surplus by 2007. Then, Oregon decided to expand its OHP Standard program by offering about 10,000 spots using a lottery to solicit or invite applications.

Lottery winners applied for Medicaid, and those determined to be eligible received coverage. The main eligibility criteria were as follows—being 19-64 years of age; being an Oregon resident who is not otherwise eligible for public insurance; being a U.S. citizen or legal immigrant; being uninsured for the previous six months, and having income below the federal poverty level with assets not exceeding $\$ 2,000$. Of the 89,824 individuals who signed up for the lottery, 35,169 won, and about $30 \%$ of the winners enrolled in Medicaid.

When interpreting the Oregon experiment results, the low-income, uninsured population served 
by OHP Standard should be considered. Finkelstein et al. (2012) elaborates the difficulties with extrapolating Oregon's finding to other Medicaid expansions. The population served by OHP Standard is neither representative of the typical Medicaid population served by OHP Plus nor the low-income uninsured population in the United States. For example, compared to the low-income U.S. population, OHP Standard's target population has more whites (84\%), fewer Blacks (2\%), is older, and reports being in worse health (Allen et al. 2010). Also, people voluntarily signed up for the lottery. These issues do not threaten the internal validity of the Oregon experiment. However, the inherent sample selection on observed (and unobserved dimensions) suggests a crucial role for effect heterogeneity in understanding its findings.

\subsection{Data}

We use the publicly available data files from Taubman et al. (2014). The sample contains ED visit information for 24,646 individuals from 2007 to 2009. The pre-lottery period spans January 1, 2007, through March 9, 2008. The study period is 18 months from the earliest notification date on March 10, 2008, through September 30, 2009. Medicaid coverage is constructed from state administrative records and defined as any receipt during the study period.

We analyze fourteen ED visit measures. These variables measure overall ED use and three categories of ED visits. The first category groups ED use by hospital admission, including outpatient and inpatient ED visits. The second category groups ED visits by the time of occurrence and consists of those occurring during on-time ( 7 am to $8 \mathrm{pm}$ on Monday to Friday) and off-time hours (nights and weekends).

The final category groups ED visits by whether they required immediate care or not. We adopt the classification of ED visits based on the primary ICD-9 diagnosis code (Taubman et al. 2014, Billings et al. 2000). While we refer the reader to Taubman et al. (2014) for a detailed description, the algorithm assigns to each ED visit a probability that it is one of four types. The first type—emergent, non-preventable—includes unpreventable illnesses requiring immediate ED care (e.g., heart attacks and nonspecific chest pain). The second type—emergent, preventable—includes 
ED visits that require immediate ED care but are avoidable (e.g., asthma attacks and urinary tract infections). The third type-primary care treatable-includes ED visits requiring immediate care but not through the emergency department (e.g., sprains, strains, and abdominal pain). The final type-non-emergent—contains ED visits that do not require immediate care (e.g., headaches and back problems). Each visit is assigned a probability of being in all four categories. The number of visits for each type is then obtained by summing the assigned probabilities across all visits (Taubman et al. 2014). We do not analyze unclassified visits not assigned to any of the above four categories.

We analyze both the binary and continuous versions of these types of ED visits. While Taubman et al. (2014) only analyzes the continuous versions, we create their binary counterparts as follows. Since the number of ED visits for each type is computed as a sum of probabilities, we conservatively classify the individual as having only one type of visit for people with one ED visit, which is set equal to the visit type with the highest probability. For those with two ED visits, we classify the individual as having at most two types of visits, that is, visit types with the top two highest probabilities. We classify the individual as having at most three visit types for those with three ED visits, with the types being those with the top three highest probabilities. Finally, we classify individuals with four or more ED visits as having all visit types with non-zero assigned probabilities.

All our analyses include variables needed to ensure unbiased estimation of treatment effects, such as household size and an indicator for which of the eight lottery draws between March and September 2008 the individual was assigned. Given our central focus on heterogeneous effects along observed dimensions, we include two additional groups of variables which have not previously being used to study effect heterogeneity in the Oregon experiment studies-lottery list variables and baseline characteristics measured before the lottery. We focus on these group of variables in exploring heterogeneity to ensure that they are not endogenous to Medicaid receipt. ${ }^{3}$

The lottery list contains eight variables constructed from the lottery sign-up sheet-age; sex; indicators for whether English is the preferred language for receiving materials; whether the individuals signed themselves up for the lottery; whether an individual provided a phone number on

${ }^{3}$ We deliberately omit other potential heterogeneity variables available from the pre-lottery survey. Those variables are only available for a small number of people, limiting the sample considerably and potentially subject to attrition bias. 
the sign-up form; whether the individuals listed their address as a P.O. Box; whether the individual signed up on the first day of the lottery, and the median household income in the applicant's zip code from the 2000 decennial census. We are only missing the last variable compared to the original analysis because it is not publicly available, but this does not affect the internal validity of the treatment effects.

The baseline characteristics are various pre-lottery ED use measures, participation in the Supplemental Nutrition and Assistance Program (SNAP), and receiving TANF in the pre-lottery period. We include twenty pre-lottery ED measures covering overall ED use, on-/off-time use, ED visits resulting in hospital admission, visits for each type described above, and visits for specific types of injuries and health conditions. We include this expanded list of pre-lottery ED visit information to capture historical emergency care demand. The pre-lottery SNAP/TANF participation variables, which proxy for one's experience with the social safety net system, come from state administrative data. Those variables include indicators of program receipt and the total household benefit amounts received between January 1, 2007, through the individual's notification date.

We restrict the sample to those with pairwise non-missing observations in the covariates and each outcome. Our baseline sample consists of 24,615 individuals with non-missing information on all covariates (i.e., 99.9 percent of Taubman et al.'s (2014) sample). We use the baseline sample to analyze our primary outcome-whether the participant had any ED visits. The analysis samples for other outcomes are slightly smaller due to missing data. However, the difference is negligible; the smallest sample comprises 24,588 observations-only 27 fewer people relative to the baseline sample. Our results are unaffected by conditioning on a uniform sample of non-missing observations across all outcomes.

Table 1 presents the summary statistics for our baseline sample. The sample is 55 percent female with an average age of 40 years. About 54 percent of the sample received SNAP with an average SNAP benefit amount of $\$ 1,332$ in the pre-lottery period. TANF receipt is much lower at 2 percent of the sample with an average benefit of \$96. The sample averaged $0.77 \mathrm{ED}$ visits in the pre-lottery 
Table 1: Descriptive Statistics

\begin{tabular}{|c|c|c|c|c|c|}
\hline Variable & Mean & SD & Median & Min & Max \\
\hline \multicolumn{6}{|l|}{ Lottery list and baseline characteristics } \\
\hline Age (years) & 39.60 & 12.05 & 39.0 & 20 & 63 \\
\hline Gave phone number & 0.87 & 0.34 & 1.0 & 0 & 1 \\
\hline English as preferred language & 0.86 & 0.34 & 1.0 & 0 & 1 \\
\hline Female & 0.55 & 0.50 & 1.0 & 0 & 1 \\
\hline Week of lottery sign up & 1.58 & 1.62 & 1.0 & 0 & 5 \\
\hline Provided P.O. box address & 0.03 & 0.16 & 0.0 & 0 & 1 \\
\hline Signed up self for lottery & 0.90 & 0.30 & 1.0 & 0 & 1 \\
\hline Pre-lottery SNAP recipient & 0.54 & 0.50 & 1.0 & 0 & 1 \\
\hline Pre-lottery SNAP benefit amount (\$) & 1331.94 & 1863.76 & 522.5 & 0 & 20745 \\
\hline Pre-lottery TANF recipient & 0.02 & 0.15 & 0.0 & 0 & 1 \\
\hline Pre-lottery TANF benefit amount $(\$)$ & 96.16 & 694.70 & 0.0 & 0 & 16031 \\
\hline \multicolumn{6}{|l|}{ Pre-randomization ED use } \\
\hline Number of overall visits & 0.77 & 1.86 & 0.0 & 0 & 17 \\
\hline Number of inpatient visits & 0.09 & 0.41 & 0.0 & 0 & 6 \\
\hline Number of outpatient visits & 0.69 & 1.71 & 0.0 & 0 & 16 \\
\hline Number of on-hours visits & 0.45 & 1.23 & 0.0 & 0 & 13 \\
\hline Number of off-hours visits & 0.33 & 0.92 & 0.0 & 0 & 10 \\
\hline Number of emergent, non-preventable visits & 0.16 & 0.50 & 0.0 & 0 & 9 \\
\hline Number of emergent, preventable visits & 0.06 & 0.28 & 0.0 & 0 & 6 \\
\hline Number of primary care treatable visits & 0.27 & 0.75 & 0.0 & 0 & 12 \\
\hline Number of non-emergent visits & 0.16 & 0.58 & 0.0 & 0 & 12 \\
\hline Number ambulatory-care-sensitive visits & 0.05 & 0.30 & 0.0 & 0 & 5 \\
\hline Number of visits (chronic conditions) & 0.14 & 0.64 & 0.0 & 0 & 9 \\
\hline Number of visits (injury) & 0.17 & 0.56 & 0.0 & 0 & 6 \\
\hline Number of visits (skin conditions) & 0.05 & 0.31 & 0.0 & 0 & 5 \\
\hline Number of visits (abdominal pain) & 0.04 & 0.27 & 0.0 & 0 & 5 \\
\hline Number of visits (back pain) & 0.03 & 0.26 & 0.0 & 0 & 5 \\
\hline Number of visits (chest pain) & 0.02 & 0.18 & 0.0 & 0 & 3 \\
\hline Number of visits (headache) & 0.02 & 0.23 & 0.0 & 0 & 4 \\
\hline Number of visits (mood disorders) & 0.02 & 0.23 & 0.0 & 0 & 5 \\
\hline Number of visits (psychiatric conditions) & 0.06 & 0.38 & 0.0 & 0 & 6 \\
\hline Sum of total ED charges & 894.85 & 2593.46 & 0.0 & 0 & 42315 \\
\hline \multicolumn{6}{|l|}{ ED use outcomes } \\
\hline Any overall visit & 0.34 & 0.47 & 0.0 & 0 & 1 \\
\hline Any inpatient visit & 0.07 & 0.26 & 0.0 & 0 & 1 \\
\hline Any outpatient visit & 0.32 & 0.47 & 0.0 & 0 & 1 \\
\hline Any emergent, non-preventable visit & 0.16 & 0.37 & 0.0 & 0 & 1 \\
\hline Any emergent, preventable visit & 0.10 & 0.30 & 0.0 & 0 & 1 \\
\hline Any primary care treatable visit & 0.22 & 0.41 & 0.0 & 0 & 1 \\
\hline Any non-emergent visit & 0.16 & 0.37 & 0.0 & 0 & 1 \\
\hline Number of overall visits & 1.00 & 2.41 & 0.0 & 0 & 22 \\
\hline Number of inpatient visits & 0.11 & 0.53 & 0.0 & 0 & 7 \\
\hline Number of outpatient visits & 0.89 & 2.21 & 0.0 & 0 & 21 \\
\hline Number of emergent, non-preventable visits & 0.21 & 0.69 & 0.0 & 0 & 14 \\
\hline Number of emergent, preventable visits & 0.07 & 0.35 & 0.0 & 0 & 8 \\
\hline Number of primary care treatable visits & 0.35 & 0.96 & 0.0 & 0 & 16 \\
\hline Number of non-emergent visits & 0.20 & 0.70 & 0.0 & 0 & 13 \\
\hline
\end{tabular}

Notes: This table presents descriptive statistics based on the Oregon Health Insurance Experiment data. The sample consists of 24,615 individuals in the Taubman et al. (2014) sample with nonmissing information on pre-lottery emergency department utilization and SNAP/TANF receipt. The pre-randomization SNAP and TANF benefits are total household benefits received from January 2007 through the individual's lottery notification date. The pre-randomization ED use measures are with respect to utilization between January 1, 2007 and March 9, 2008. 
period, with the total emergency department facility charges being $\$ 895$ on average. ${ }^{4}$

\section{Methods}

Our analysis of the impacts of Medicaid on ED use is twofold. First, we estimate different types of heterogeneous effects of Medicaid on ED utilization using generalized random forests (GRF). GRF is an extension of random forests that can estimate various population quantities identified as solutions to local moment conditions (Wager \& Athey 2018, Athey et al. 2019). In the second part of our analysis, we use the heterogeneous treatment effects to estimate assignment rules using policy learning algorithms (Athey \& Wager 2021, Kitagawa \& Tetenov 2018).

\subsection{Treatment effect heterogeneity using generalized random forests}

We represent the treatment effect parameters in the potential outcomes framework. Let $D \in$ $\{0,1\}$ denote the individual's Medicaid coverage. Also, let $Y(D)$ represent the potential outcomes associated with Medicaid receipt $(D=1)$ or otherwise $(D=0)$. Since Medicaid coverage is endogenous, we pursue instrumental variable estimation using Oregon's lottery as an instrument, denoted by $Z \in\{0,1\}$. As such, let $D(Z)$ be the potential treatment status corresponding with the instrument, with $D(1)$ being the potential treatment with winning the lottery and $D(0)$ denoting the potential treatment with losing the lottery. Suppose that the outcome is given by a structural model of the form $Y=\mu(X)+\tau(X) D+\varepsilon$, where $X$ is a vector of covariates, $\mu(X)$ is the mean outcome, $\tau(X)$ is the heterogeneous treatment effect, and $\varepsilon$ is the error term such that $\mathbb{E}[\varepsilon \mid X, Z]=0$.

Abadie (2003) shows that $\tau(X)$ can be expressed as the simple IV estimand, which identifies the conditional LATE (i.e., the conditional average treatment effect for the sub-population of

${ }^{4}$ The continuous measures of ED utilization have been top-coded in the public-use Oregon experiment files such that each value has as least ten observations. Nonetheless, this does not matter practically given Taubman et al.'s (2014) online appendix states that "[t]his means that for many of the number-of-visit outcomes, the publicly available data will not directly replicate the results presented in the main text and supplementary materials, although our findings are robust to the censoring we imposed in the public use data." 
compliers): ${ }^{5}$

$$
\tau(X)=\frac{\mathbb{E}[Y \mid X, Z=1]-\mathbb{E}[Y \mid X, Z=0]}{\mathbb{E}[D \mid X, Z=1]-\mathbb{E}[D \mid X, Z=0]}=\mathbb{E}[Y(1)-Y(0) \mid X, D(1)=1, D(0)=0]
$$

As captured by $\tau(X)$, the heterogeneous effects with respect to covariates refers to various types of conditional average treatment effects (CATE) for compliers at different covariate levels. ${ }^{6}$ At the most granular level, $\tau(X)$ is an estimate of the individualized treatment effect (ITE) in the smallest covariate partition defined by an individual's covariate values. At higher levels, $\tau(X)$ represents the average treatment effect for well-defined subgroups. We refer to such aggregated parameters as the group average treatment effects (GATE) (Lechner et al. 2020). Several machine learning methods for causal inference have been proposed for estimating heterogeneous treatment effects. For a recent review, see Knaus et al. (2021).

In this paper, we estimate $\tau(X)$ using generalized random forests (Athey et al. 2019). To motivate how the forest-based estimation works, consider the case of a completely randomized experiment where we are interested in the CATE for some specified covariates. Wager \& Athey (2018) proposed causal forest for this scenario. ${ }^{7}$ The basic building block of causal forests is a causal tree. Causal trees recursively split the sample into small leaves, $L(x)$, for any given point $x$ defined by the vector of covariates. For any leaf, we can then estimate the CATE as the difference in means between treatment and control units. The causal forest is then an average of several such causal trees.

While causal forests are appealing, they do not work for observational studies such as those relying on instrumental variables. Athey et al. (2019) introduced generalized random forests as an

${ }^{5}$ See Proposition 5.3 in Abadie (2003). The identification result are based on the standard assumptions needed for the IV estimand to identify the LATE_-instrument independence, exclusion of the instrument, relevance of the instrument and monotonicity (Imbens et al. 1994, Angrist et al. 1996, Abadie 2003).

${ }^{6}$ For easier exposition, we refer to $\tau(X)$ as CATE in the rest of the paper although a more appropriate acronym is CLATE when performing IV estimation.

${ }^{7}$ Causal forests are an extension of the idea of random forests to causal inference problems (Breiman 2001). Traditional random forests aim to predict an outcome based on some observed covariates. Random forests are, in turn, an average of the so-called regression trees for predicting a continuous outcome. A regression tree is built by splitting the sample into partitions to minimize the mean-squared error of predictions. After recursively partitioning the sample into a tree structure, the predictions are obtained as the outcome means in each leaf. Each tree is grown on a random sub-sample and a random subset of the variables. Since regression trees are unbiased but exhibit high variance, random forests average the final predictions across several regression trees, leading to stable forest-based predictions. For an introduction to random forests for prediction tasks, see Hastie et al. (2009). 
adaptive nearest-neighbor estimator that solves a locally weighted version of a population moment condition. As such, causal forests can be viewed as a special type of generalized random forests. In the case of estimating the conditional LATE, generalized random forests apply because we can view $\tau(X)$ as a parameter identified by the population moment conditions:

$$
\mathbb{E}\left[\left(Y_{i}-\mu(X)-\tau(X) D_{i}\right)\left(1 Z_{i}^{\prime}\right)^{\prime} \mid X_{i}=x\right]=0
$$

To proceed with estimation, Athey et al. (2019) first define a similarity weight, $\alpha_{i}(X)$, which captures the importance of unit $i$ to estimating $\tau(X)$ at point $x$. The estimates $(\widehat{\tau}(x), \widehat{\mu}(x))$ are then the solutions to the local estimating equations based on (2):

$$
\sum_{1}^{n} \alpha_{i}(x)\left[\left(Y_{i}-\widehat{\mu}(x)-\widehat{\tau}(x) D_{i}\right)\left(1 Z_{i}^{\prime}\right)^{\prime}\right]=0 .
$$

Part of the innovation in Athey et al. (2019) is to use random forests to estimate the weight, $\alpha_{i}(X)$, needed for the solution to the heterogeneous estimating equation in (3). The weights are obtained by first growing $B$ trees using the standard regression tree algorithm. However, unlike traditional regression trees, the splits at the parent nodes are chosen to maximize heterogeneity in $\tau(X)$. For each tree, the observations that fall in the same leaf as $x$ are marked and given a positive weight. The forest-based algorithm then averages over the $B$ tree-based neighborhoods to obtain $\alpha_{i}(X)$, which are then used to fit the estimating equations in (3). Thus, the similarity weight $\alpha_{i}(X)$ measures how often an observation falls in the same neighborhood (leaf) as $x$. Athey et al. (2019) show that using the forest-based weights in equation (3) yields consistent and asymptotically normal estimates of $\tau(X)$.

We note some key implementation details. We grow all forests using 100,000 trees and randomly sample half the data to build each tree. Tree building relies on further subsample splitting based on the honesty principle, which is the idea that different sub-samples should be used to determine tree splits and estimate the within-leaf treatment effects to reduce bias (Wager \& Athey 2018). Here, we use half of the sampled data to determine the tree splits and the other half for estimation. For each 
randomly drawn subsample, we must also randomly select some covariates to build each tree. We use the default rule-of-thumb to determine the number of variables considered at each split point. That is, if $p$ is the number of covariates, then the number of variables used in each split is the minimum of $(\sqrt{p}+20)$ and $p$. For inference, the variance of the forest-based $\widehat{\tau}(X)$ is constructed using the bootstrap of little bags, which amounts to training trees in small groups and comparing $\widehat{\tau}(X)$ predictions within and across those groups (Athey et al. 2019, Sexton \& Laake 2009). We cluster standard errors at the household level.

To quantify which covariates are most important for driving treatment effect heterogeneity in growing the forest, we report a variable importance ranking of all covariates used in growing the forest. This variable importance measure is based on the number of times the variable is used for splitting, weighted by the tree depth of the split. In addition to the nonparametric estimates of $\tau(X)$, we can obtain average treatment effects by averaging the individualized treatment effects. We plug the $\tau(X)$ estimates into the standard augmented inverse probability weighting (AIPW) estimator to obtain more accurate average estimates. Doing so allows the average estimates to retain the desirable doubly robust property (Chernozhukov et al. 2018). Similarly, we can obtain doubly robust GATE estimates for specific subgroups defined by one or more covariates.

\subsection{Policy learning exercises}

Estimating heterogeneous treatment effects opens the doorway for performing interesting policy learning exercises that help understand alternative treatment assignment regimes and target program recipients with educational interventions. We perform two exercises leveraging the estimated heterogeneous effects in the intent-to-treat (ITT) scenario. We focus on the ITT context for the policy learning exercises because it more closely aligns with the practical problem of allocating Medicaid spots in Oregon's expansion of OHP Standard to a new population. Both exercises draw heavily on the literature on statistical decision rules that uses the distribution of treatment effects to design treatment assignment rules that maximize mean social welfare, typically defined as the mean post-treatment outcome (Manski 2004, Dehejia 2005, Hirano \& Porter 2009, Stoye 2009, 2012, 
Kitagawa \& Tetenov 2018, Athey \& Wager 2021).

The first exercise is a theoretical one that estimates an alternative treatment assignment scheme selecting whom to solicit applications from to achieve a well-defined objective. This alternative treatment assignment scheme is necessarily infeasible because state Medicaid administrators cannot use it in practice to solicit applications. However, we estimate the alternative assignment scheme to contrast those selected under it with the observed random assignment scheme in the data. The question we seek to answer here is, how should a social planner who wants to optimize some objective function (e.g., minimize unnecessary ED use) optimally choose people to treat (i.e., solicit applications from in the Oregon context), and how would the assigned population differ from the lottery population?

In this exercise, we compute the optimal allocation scheme based on a specified objective function to solicit Medicaid applications using integer programming (Kitagawa \& Tetenov 2018). The integer programming approach lets us incorporate capacity constraints, allowing us to restrict the selected sample to equal the number of lottery winners in the Oregon experiment. We then compare the population selected based on the optimal linear program to those who won the Oregon lottery.

The second exercise is more practical and aims to identify enrollees at risk of increased ED utilization that may be deemed unnecessary by administrators. As mentioned above, states are constantly exploring policy options to curb the non-emergent use of emergency departments by Medicaid recipients. In this case, how would a simple decision rule identifying recipients most likely to increase unnecessary ED visits look? We use Athey \& Wager's (2021) policy learning algorithm to estimate a simple depth-2 (shallow) decision tree. Administrators can use such decision rules to select and target enrollees (who already received Medicaid) for specific educational interventions.

Estimating the assignment rules requires calculating doubly robust scores targeting the estimand of interest-the ITT effect of winning the lottery effect in this case. Then we choose a policy $\pi$ in the finite-depth class of policies $\Pi$ to minimize regret, i.e., the loss resulting from not choosing the ideal policy (Athey \& Wager 2021). Previous work has shown that this optimization problem 
is equivalent to a weighted binary classification problem, effectively predicting the sign of the treatment effect, where the weights are given by the size of the conditional treatment effects (e.g. Zhao et al. 2012).

The above policy learning exercises require three practical choices. The first is to define the post-treatment outcome or utility constituting the objective function. We use the number of nonemergent ED visits described in the data section. Such visits do not require immediate care and are most likely deemed unnecessary (i.e., not requiring service in the ED) by state administrators.

Second, we choose which covariates to include in the decision rule estimation. We exclude variables that interfere with fairness, ethical and legal considerations. While we use the complete set of covariates to estimate the heterogeneous treatment effects, we limit the variables used to estimate the assignment rules to nine pre-lottery ED utilization variables. Specifically, we include the overall number of pre-lottery ED visits, the number of inpatient ED visits, the number of outpatient ED visits, the number of on-hour ED visits, and the number of off-hours ED visits. The remaining variables indicate the type of pre-lottery ED utilization-indicators of any pre-lottery emergent ED use, any pre-lottery primary care treatable ED use, any pre-lottery non-emergent ED use, and the pre-lottery sum of total ED facility charges. Finally, we estimate the assignment rules as decision trees of depth 2. Since the algorithm obtains the decision rule by exact (exhaustive) tree search, the required time for estimating more complex decision rules is exponential in tree depth.

\section{Results}

\subsection{Distribution of individualized treatment effects of Medicaid on ED use}

We first present the heterogeneous impacts of Medicaid using generalized random forests as described in Section 3. We mainly focus on the extensive margin results of receiving Medicaid on binary indicators of ED use in the paper. We briefly refer to additional intensive margin results of Medicaid on continuous ED visit measures in Appendix A. Also, we provide the intent-to-treat heterogeneous effects of winning the lottery on ED utilization in Appendix B. 
Figure 1 displays the individualized treatment effects of Medicaid on the probability of any visit, with the darker blue shade indicating statistical significance at the $10 \%$ level. The subplots in Figure 1 display results for any overall visits (panel a), any outpatient visits (panel b), and any inpatient visits (panel c). These results come from $\tau(X)$ estimates at the smallest covariate partition, with each point $X$ denoting each individual's covariate values. Table 2 complements the graphical results by reporting selected quantiles of the empirical distribution of the ITE estimates for all outcomes. Table 2 also reports the average effect in the first column (discussed momentarily in Section 4.2) and the share of the heterogeneous effects that are non-positive in the last column.

We find substantial and meaningful heterogeneous effects of Medicaid across all ED visit outcomes. Specifically, for any ED visits, the heterogeneous effects of Medicaid span a wide range of negative and positive values. Although the average effect of Medicaid turns to be positive, about one-third of the ITE estimates are negative, with the $25^{\text {th }}$ percentile being a reduction of 3.1 percentage points. Moreover, there is considerable variation in the magnitude of the of ITE estimates when we focus on the positive heterogeneous effects. For instance, Table 2 shows that the median heterogeneous effect is a 7 percentage points increase, which is larger than the mean effect of 4.5 percentage points.

In addition, the statistically significant increases in ED use at the $10 \%$ level are limited to about $14.2 \%$ of the sample (with $8 \%$ being significant at the $5 \%$ level). Interestingly, there is no overlap between the statistically significant ITE estimates and the overall mean effect, further suggesting that the average effect masks heterogeneity. To put this non-overlapping finding in context, at the $10 \%$ level, the smallest ITE estimate among those who significantly increase ED usage is 4.9 percentage points, about $10 \%$ larger then the mean effect. ${ }^{8}$ When we incorporate statistical significance in discussing the heterogeneous effects, we continue to find evidence of decreases in ED use. While most of the significant ITE estimates are positive, we find that about $2 \%$ of all individualized effects are statistically significant reductions in ED use.

${ }^{8}$ At the 5\% level, the smallest ITE estimate among those who significantly increase ED usage is 7.6 percentage points, close to 1.7 times the mean effect. 
Figure 1: Distribution of individualized treatment effects of Medicaid on the propensity of any ED visit

(a) Any visit

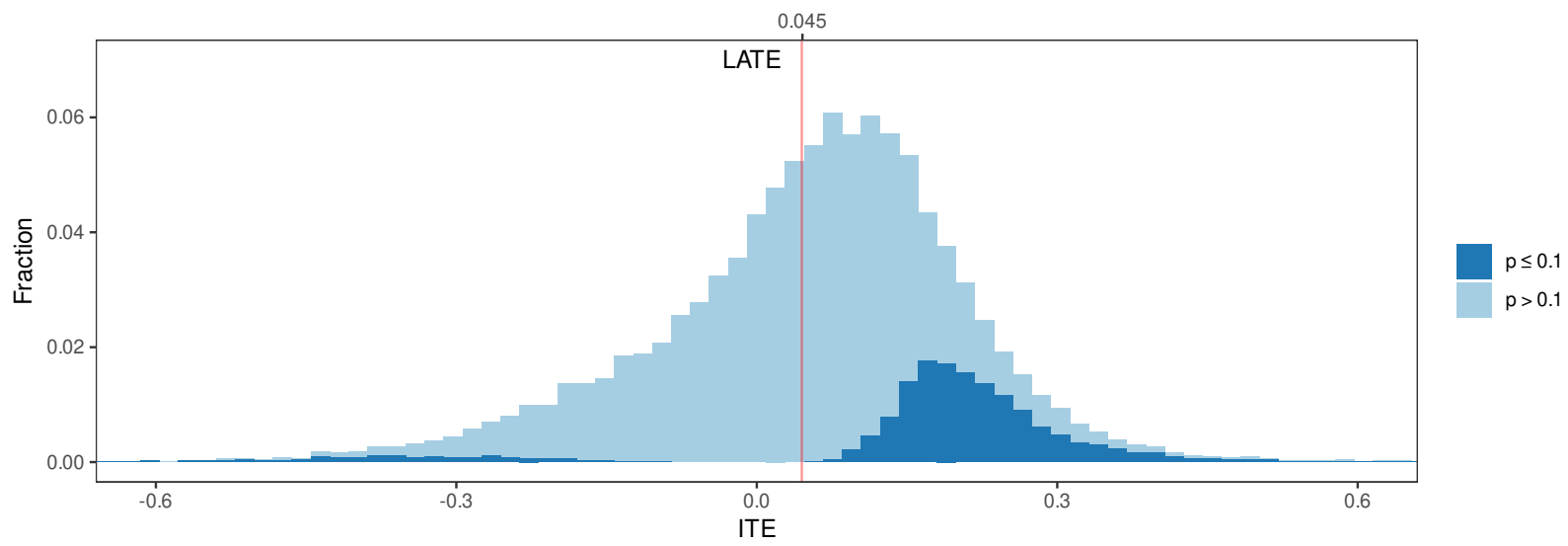

(b) Outpatient

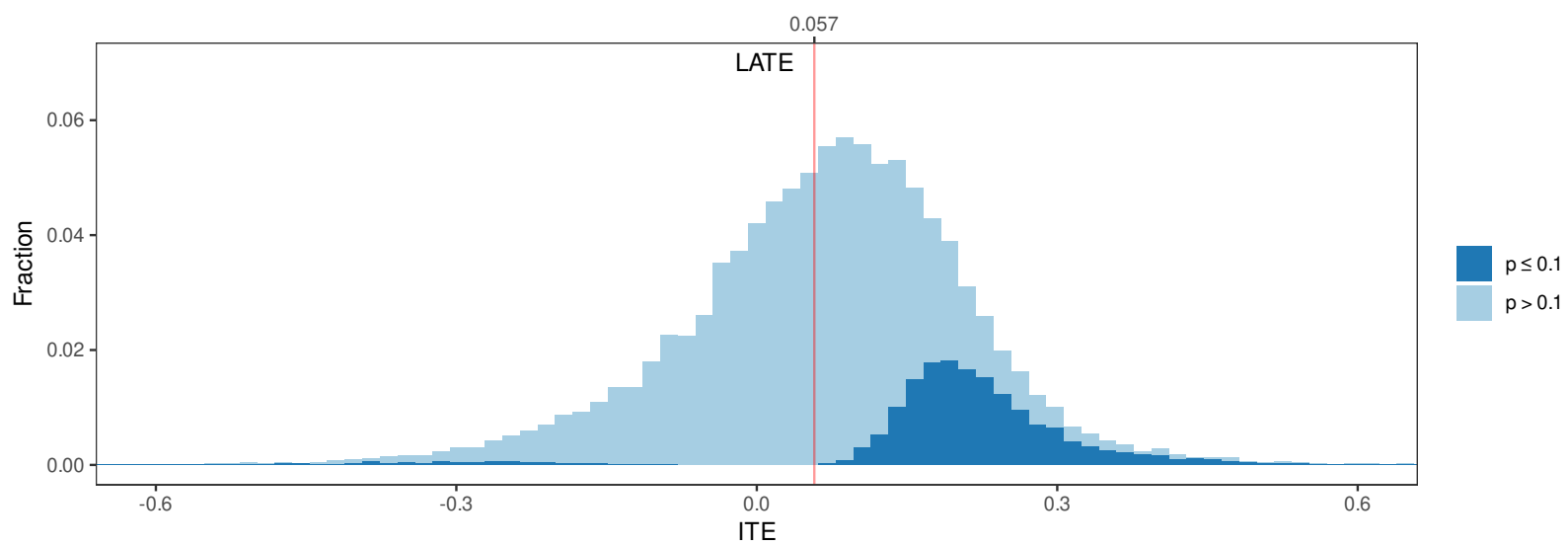

(c) Inpatient

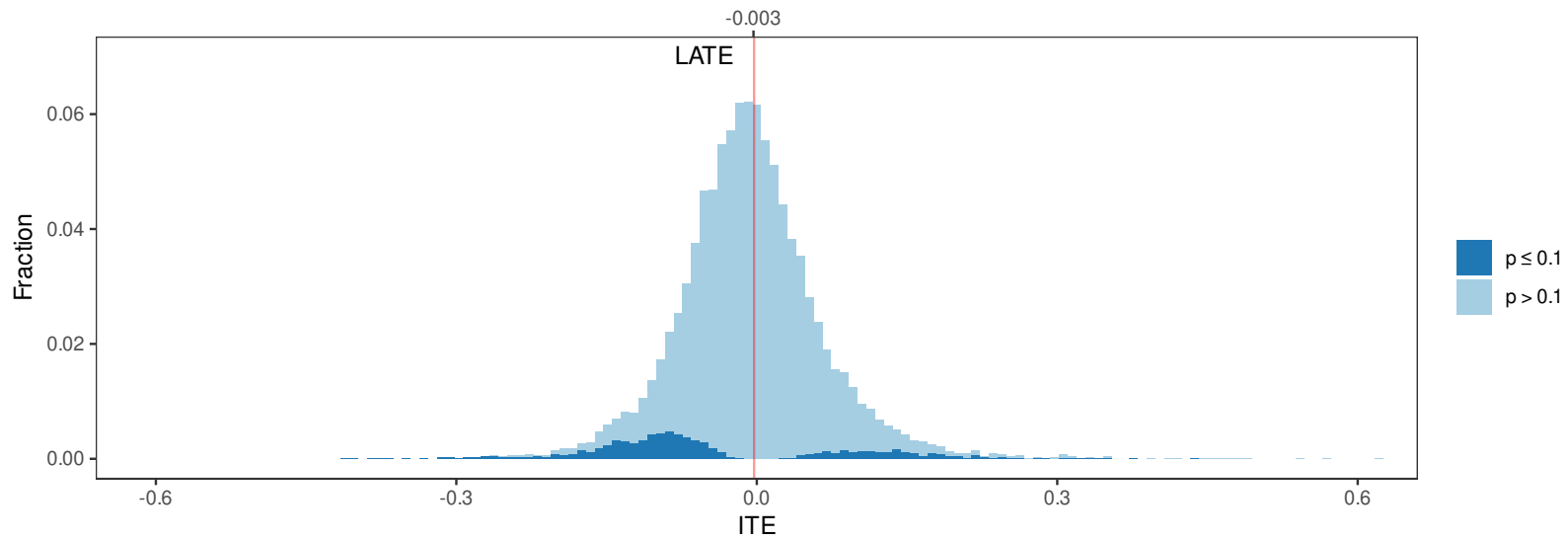

Notes: This figure plots the individualized treatment effects of Medicaid on any overall ED visit (panel a), any outpatient ED visits (panel b), and any inpatient ED use (panel c) based on generalized random forests. The darker shade denotes statistical significance at the $10 \%$ level. The red vertical line indicates the local average treatment effect. The baseline sample consists of 24,615 individuals in the Taubman et al. (2014) sample with non-missing information on pre-lottery emergency department utilization and SNAP/TANF receipt. The estimates displayed exclude less than half a percentile at the top and bottom of the distribution, resulting in the axes corresponding approximately to the percentile range $[0.5 \%, 99.5 \%]$. Bin size is chosen according to the Freedman-Diaconis rule. 
Table 2: Empirical quantiles of the distribution of individualized treatment effects of Medicaid on ED use

\begin{tabular}{lrrrrrrr}
\hline Variable & LATE & Min & $25 \%$ & $50 \%$ & $75 \%$ & Max & Share non-pos. \\
\hline Extensive margin & & & & & & & \\
$\quad$ Any overall visit & 0.045 & -1.122 & -0.031 & 0.070 & 0.151 & 0.894 & 0.31 \\
$\quad$ Any inpatient visit & -0.003 & -0.415 & -0.049 & -0.010 & 0.031 & 0.619 & 0.57 \\
$\quad$ Any outpatient visit & 0.057 & -1.121 & -0.013 & 0.078 & 0.160 & 0.800 & 0.28 \\
$\quad$ Any emergent, non-preventable visit & -0.007 & -0.622 & -0.075 & 0.003 & 0.072 & 0.583 & 0.49 \\
Any emergent, preventable visit & 0.035 & -0.313 & -0.014 & 0.030 & 0.077 & 0.532 & 0.32 \\
Any primary care treatable visit & 0.048 & -0.813 & -0.011 & 0.067 & 0.141 & 0.785 & 0.28 \\
$\quad$ Any non-emergent visit & 0.073 & -0.607 & -0.004 & 0.060 & 0.132 & 0.604 & \\
Intensive margin & & & & & & & \\
$\quad$ Number of overall visits & 0.354 & -1.503 & 0.006 & 0.273 & 0.567 & 5.939 & \\
$\quad$ Number of inpatient visits & -0.012 & -0.941 & -0.074 & -0.013 & 0.046 & 1.072 & 0.24 \\
$\quad$ Number of outpatient visits & 0.360 & -1.199 & 0.052 & 0.291 & 0.571 & 5.828 & 0.56 \\
$\quad$ Number of emergent, non-preventable visits & 0.028 & -0.590 & -0.066 & 0.020 & 0.109 & 3.025 & 0.20 \\
$\quad$ Number of emergent, preventable visits & 0.038 & -1.533 & -0.015 & 0.026 & 0.077 & 0.724 & 0.44 \\
$\quad$ Number of primary care treatable visits & 0.161 & -0.550 & 0.018 & 0.131 & 0.255 & 3.689 & 0.34 \\
$\quad$ Number of non-emergent visits & 0.107 & -0.663 & -0.008 & 0.061 & 0.143 & 2.606 & 0.22 \\
\hline
\end{tabular}

Notes: This table reports selected quantiles of the individualized treatment effects of Medicaid on ED use based on generalized random forests. The first column reports the average effect. The final column reports the share of ITEs that are non-positive. The baseline sample consists of 24,615 individuals in the Taubman et al. (2014) sample with non-missing information on pre-lottery emergency department utilization and SNAP/TANF receipt.

An important distinction when examining ED visits is whether they are for outpatient or inpatient care since the latter signifies visits for conditions severe enough to warrant hospitalization. Regarding average effects, Taubman et al. (2014) find a statistically significant effect for outpatient visits and null effects for inpatient ED use. Both types of ED visits exhibit substantial effect heterogeneity, but there are some crucial differences. A comparison of panels (a) and (b) in Figure 1 reveals that Medicaid's impacts on ED utilization are concentrated among outpatient visits. Moreover, the pattern of the distribution of ITE estimates for outpatient visits is similar to any overall ED visit, with negative and positive values. For instance, the $25^{\text {th }}$ percentile for outpatient visits is a reduction of 1.3 percentage points. However, the median ITE estimate of 7.8 percentage points is larger than the mean effect of 5.7 percentage points. Again, we find that the statistically significant increases in outpatient ED use are concentrated in the right tail of the distribution. About $16 \%$ of all estimated outpatient effects are positive and statistically significant, compared to $0.8 \%$ which are negative and significant.

For inpatient ED use, the distribution of the individualized treatment effects is relatively tighter 
than those for any ED or outpatient ED use. Notably, the distribution of the ITE estimates shows that the null effects of Medicaid on inpatient ED use mask an important heterogeneity. Panel (c) of Figure 1 shows sizable proportions of statistically significant negative and positive effects, with a slightly bigger mass for the negative ITEs. Thus, Medicaid coverage appears to have significantly reduced inpatient ED use for a reasonable share of participants. In contrast to outpatient visits, statistically significant and negative effects exceed significant and positive effects (6\% vs. $2 \%)$. The significant positive and negative heterogeneous effects appear to have counterbalanced and produced a null effect on average.

For the intensive margin, we obtain the same pattern of results for the number of ED visits. The corresponding graphs for the number of visits are shown in Figure A.1 of Appendix A, with Table 2 showing selected quantiles of the ITE distribution. Again, we find meaningful heterogeneity with individualized treatment effects ranging from a reduction of 1.50 to an increase of 5.94 ED visits. Unlike the extensive margin, the median of 0.27 is slightly smaller than the mean effect of $0.35 \mathrm{ED}$ visits. However, we still find a concentration of statistically significant increases in the number of ED visits in the distribution's right tail. On the intensive margin, we also see the similarities and differences mentioned above for the outpatient and inpatient care. First, the results for outpatient ED visits closely mirror the overall number of ED visits. The outpatient ITE estimates span a decrease of 1.20 to an increase of 5.83 ED visits, with the median and mean effects being 0.29 and 0.36 , respectively. Second, the inpatient ITE estimates are more tightly distributed. The distribution has substantially more mass to the left, outweighing the effect of high-use individuals in the right tail, which results in a statistically insignificant mean effect.

We turn our attention to the heterogeneous results by type of ED visit presented in Figure 2 for the extensive margin. Previous studies have highlighted the importance of looking beyond overall ED visits and categorizing them by medical urgency (Taubman et al. 2014, Giannouchos et al. 2022, Garthwaite et al. 2019). Consistent with the overall ED visit results, individualized treatment effects of Medicaid for all types of ED visits exhibit substantial heterogeneity. All ED visit types show a wide range of ITE estimates, including negative and positive values. Moreover, the share 


\section{Figure 2: Distribution of individualized treatment effects of Medicaid on the propensity of any ED visit by type of condition}

(a) Emergent, non-preventable



(c) Primary care treatable

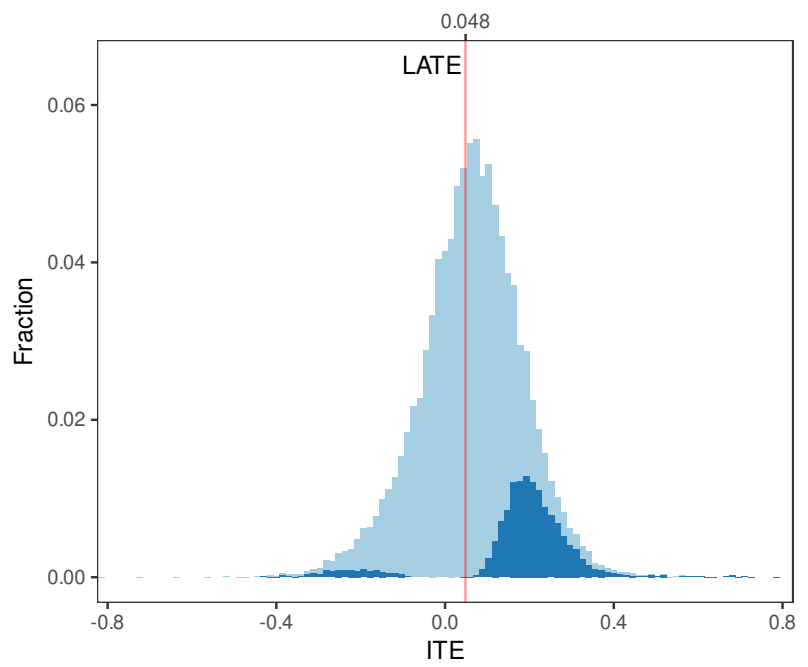

(b) Emergent, preventable

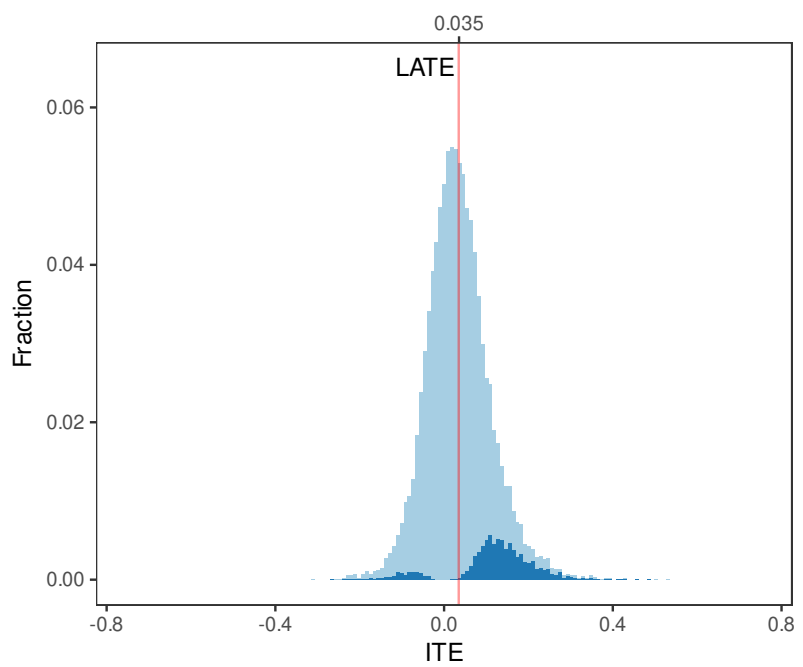

(d) Non-emergent

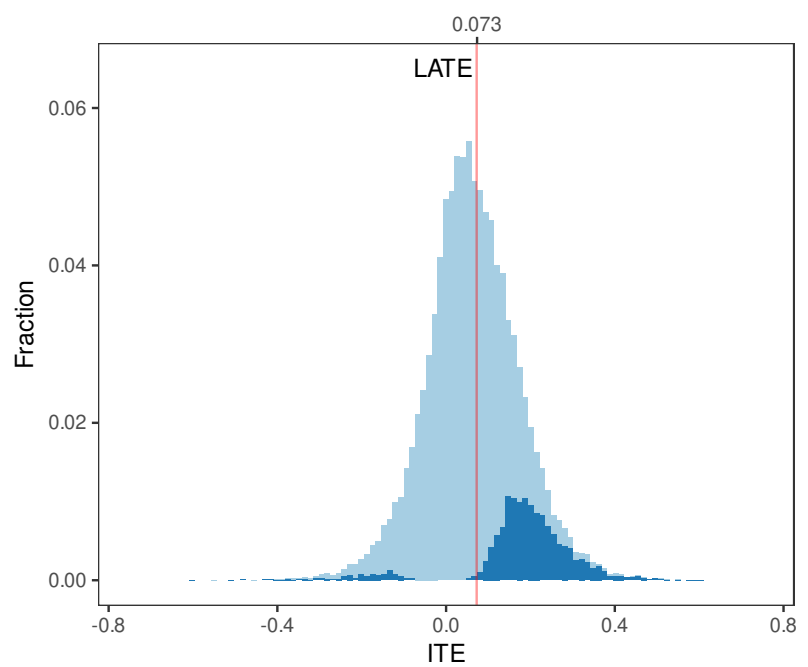

$p \leq 0.1$

$p>0.1$

Notes: This figure plots the individualized treatment effects of Medicaid by type of ED visit based on generalized random forests for any emergent, non-preventable visit (panel a), any emergent, preventable visit (panel b), any primary care treatable visit (panel c), and any non-emergent visit (panel d). Measures of the type of ED visit are based on Billings et al.'s (2000) algorithm described in Taubman et al. (2014). We use these measures to construct binary indicators of ED visits by type of condition as described in the main text. The darker shade denotes statistical significance at the $10 \%$ level. The red vertical line indicates the local average treatment effect. The baseline sample consists of 24,615 individuals in the Taubman et al. (2014) sample with non-missing information on pre-lottery emergency department utilization and SNAP/TANF receipt. The estimates displayed exclude less than half a percentile at the top and bottom of the distribution, resulting in the axes corresponding approximately to the percentile range [0.5\%, 99.5\%]. Bin size is chosen according to the Freedman-Diaconis rule. 
of significant ITE estimates differs across the ED visit types. For any emergent, non-preventable visits, panel (a) in Figure 2 shows negative and positive statistically significant effects of sizable proportions. These two opposing forces are consistent with the average null effect in Taubman et al. (2014). This finding accords with the large share of negative ITE estimates discussed above for inpatient ED care. Across all visit types, we find the largest share of significant reductions in ED uses for emergent, non-preventable visits. A similar pattern holds for the number of emergent, non-preventable visits, with the corresponding graph in Figure A.2 of Appendix A.

Figure 2 shows some differences in the heterogeneous impacts for the remaining three types of ED visits. First, we find a larger share of positive and statistically significant individualized effects for primary care treatable and non-emergent visits than emergent visits (i.e., non-preventable and preventable) on both extensive and intensive margins. The two largest increases on the extensive and intensive margins occur for primary-care treatable and non-emergent visits. However, the increase in primary care treatable visits is more pronounced on the intensive margin, whereas the increase in non-emergent visits is larger on the extensive margin.

In summarizing, the pattern of heterogeneous effects we uncover offers a more nuanced interpretation of Medicaid's effect on ED utilization that is not discernible by focusing on the mean effect alone. For instance, the large fraction of statistically significant increases for primary care treatable and non-emergent visits suggests that gaining Medicaid coverage increased ED use in these categories for most people, not just on average. In contrast, we observe a sizable amount of negative ITEs for the emergent, non-preventable category. While the average effect is not statistically significant, this finding suggests that some people reduced ED usage by not seeking treatment for conditions that may require emergency department treatment.

It is also instructive to highlight the most important covariates driving these heterogeneous effects. Put differently, what are the most used individual characteristics for tree splitting in growing the causal forests? Table 1 lists the covariates utilized in growing the causal forestspre-randomization ED use (overall use and by visit type), SNAP and TANF receipt measures, and lottery list variables. Figure 3 displays the variable importance scores for the top 20 characteristics. 
Figure 3: Variable importance scores in growing the causal forest (Any Visit)

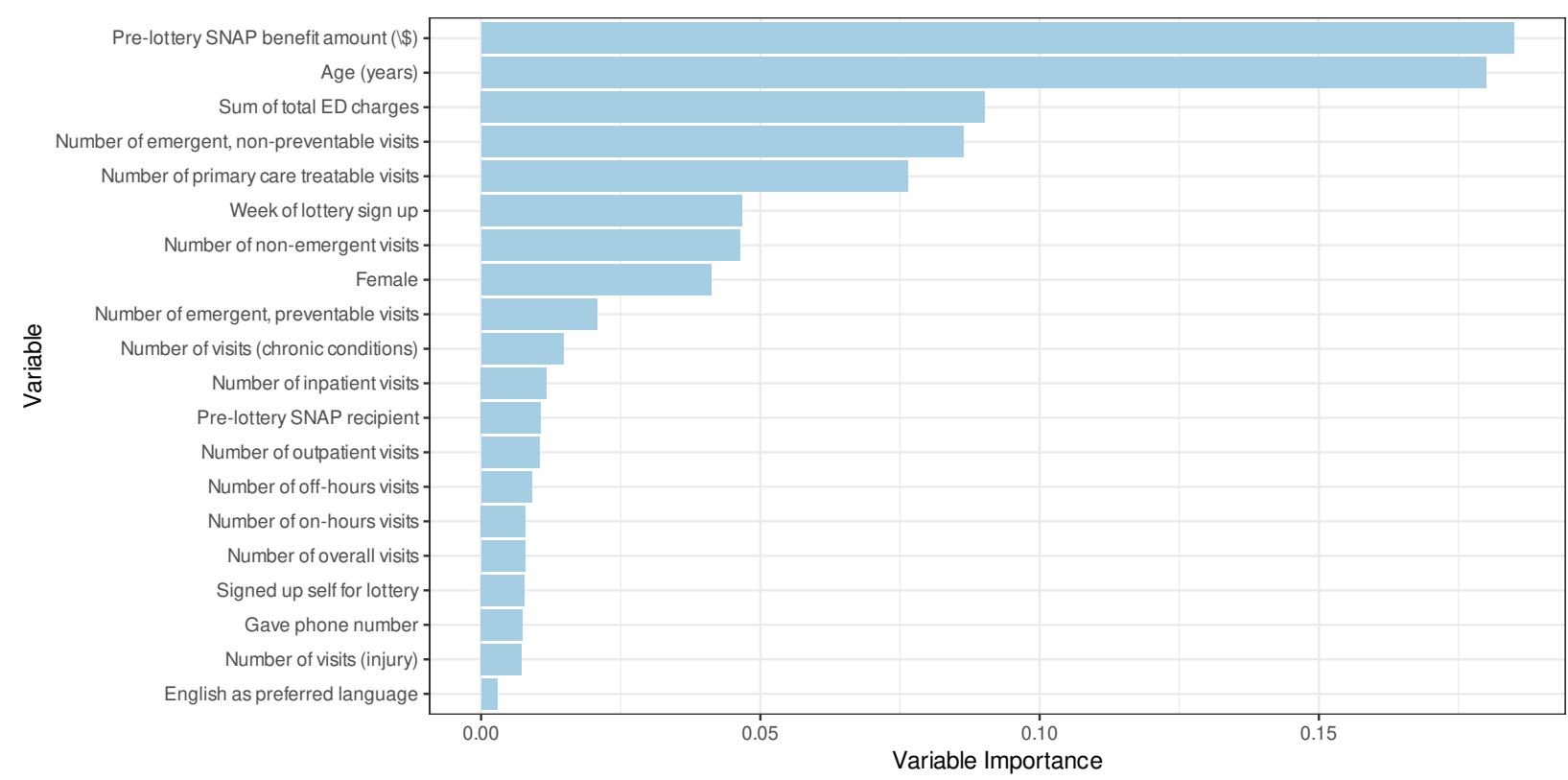

Notes: This figure shows the variable importance scores of the top 20 characteristics in growing the generalized random forests used to estimate the individualized treatment effects of Medicaid for any overall ED visit. The variable importance measure is a simple weighted sum of the proportion of times a variable is used in a tree splitting step at each depth in growing the forest. The scores roughly capture how important a variable is for driving treatment effect heterogeneity. The baseline sample consists of 24,615 individuals in the Taubman et al. (2014) sample with non-missing information on pre-lottery emergency department utilization and SNAP/TANF receipt.

We present the corresponding variable importance plot for the number of ED visits and the scores for all variables in Appendix A. For the extensive margin, Figure 3 shows that the causal forest splits the most on pre-lottery SNAP benefits followed by age, the sum of total ED facility charges, the number of pre-lottery emergent but non-preventable ED visits, and the number of pre-lottery primary care treatable ED visits.

\subsection{Aggregate effects of Medicaid on ED use}

In this section, we present doubly robust estimates of the average Medicaid effect (i.e., the LATE). Although not the main focus of our analysis, a computationally cheap by-product of estimating heterogeneous effects is that we can aggregate them to produce the average effects. These average effects are obtained by averaging the doubly robust scores targeting the LATE instead of computing a simple average of the individualized treatment effects, $\widehat{\tau}(X)$. Given that the standard linear IV 
Table 3: Treatment effect estimates of Medicaid coverage on ED use

\begin{tabular}{|c|c|c|c|c|c|c|}
\hline \multirow[b]{2}{*}{ Variable } & \multicolumn{3}{|c|}{ GRF estimates } & \multicolumn{3}{|c|}{$\begin{array}{l}\text { Linear estimates } \\
\text { (Taubman et al. (2014) Replication) }\end{array}$} \\
\hline & LATE & SE & $p$-value & LATE & SE & $p$-value \\
\hline \multicolumn{7}{|l|}{ Extensive margin } \\
\hline Any overall visit & 0.045 & 0.028 & 0.111 & 0.069 & 0.024 & 0.004 \\
\hline Any inpatient visit & -0.003 & 0.015 & 0.855 & -0.011 & 0.013 & 0.424 \\
\hline Any outpatient visit & 0.057 & 0.028 & 0.037 & 0.082 & 0.024 & 0.001 \\
\hline Any emergent, non-preventable visit & -0.007 & 0.021 & 0.743 & 0.007 & 0.019 & 0.731 \\
\hline Any emergent, preventable visit & 0.035 & 0.016 & 0.029 & 0.039 & 0.015 & 0.009 \\
\hline Any primary care treatable visit & 0.048 & 0.024 & 0.040 & 0.069 & 0.021 & 0.001 \\
\hline Any non-emergent visit & 0.073 & 0.021 & 0.000 & 0.064 & 0.019 & 0.001 \\
\hline \multicolumn{7}{|l|}{ Intensive margin } \\
\hline Number of overall visits & 0.354 & 0.114 & 0.002 & 0.375 & 0.106 & 0.000 \\
\hline Number of inpatient visits & -0.012 & 0.028 & 0.676 & -0.017 & 0.026 & 0.516 \\
\hline Number of outpatient visits & 0.360 & 0.106 & 0.001 & 0.387 & 0.098 & 0.000 \\
\hline Number of emergent, non-preventable visits & 0.028 & 0.034 & 0.414 & 0.040 & 0.032 & 0.223 \\
\hline Number of emergent, preventable visits & 0.038 & 0.018 & 0.039 & 0.036 & 0.017 & 0.034 \\
\hline Number of primary care treatable visits & 0.161 & 0.049 & 0.001 & 0.171 & 0.045 & 0.000 \\
\hline Number of non-emergent visits & 0.107 & 0.036 & 0.003 & 0.105 & 0.033 & 0.002 \\
\hline
\end{tabular}

Notes: This table reports the estimates of Medicaid coverage on ED use based on generalized random forests and a linear IV model. The sample consists of 24,615 individuals in the Taubman et al. (2014) sample with non-missing information on pre-lottery emergency department utilization and SP/TANF receipt.

estimator also recovers the LATE, we only discuss the forest-based LATE to replicate the results in Taubman et al. (2014) and show that the qualitative average findings are unchanged. ${ }^{9}$

Table 3 presents the nonparametric and linear IV estimates of Medicaid's effect on all measures of ED utilization. The linear IV estimates confirm that we closely replicate Taubman et al.'s (2014) main findings, with minor differences in magnitude plausibly due to rounding and the top-coding in the public-use Oregon data files. The fact that we replicate their findings is not surprising given the negligible difference between our samples when we restrict the sample to observations with non-missing pre-lottery utilization. ${ }^{10}$ Therefore, we are confident that our linear IV results are practically the same as those in Taubman et al. (2014) and serve as a useful baseline for comparing our nonparametric results.

Except for some differences in magnitudes in a few cases of the binary outcomes, the nonparametric LATE estimates are qualitatively the same as those based on the linear IV method. On the

${ }^{9}$ See Słoczyński (2020) for a recent discussion on when the linear IV estimand recovers the LATE when covariates are included in the IV estimation.

${ }^{10}$ In their online appendix, Taubman et al. (2014) note using "a missing indicator to handle the small number of missing prerandomization observations." 
extensive margin, the nonparametric method appears slightly inefficient (higher standard errors), but there is no difference in statistical significance between the estimates from the two methods. On the intensive margin, the nonparametric estimates more closely align with the linear method. Overall, our nonparametric estimates of the average effect comport with previous studies that Medicaid increased ED use while allowing us to explore heterogeneity at a more granular level.

\subsection{Risk factors associated with changes in ED use}

This section investigates the risk factors associated with significant changes in ED usage by examining treatment effects for selected subgroups. We summarize treatment effects for selected subgroups by aggregating the individualized treatment effects discussed above. Table 4 presents group average treatment effects for an extended list of characteristics from the causal forest.

There are two notable findings. First, we find statistically significant GATE estimates for four subgroups - men, prior SNAP participants, young and middle-aged adults below age 50, and individuals with any previous ED use classified as primary care treatable. These groups exhibit treatment effects between 8 and 10 percentage points, at least twice the average effect. If we zero in on smaller subgroups at the intersection of these characteristics, we find even larger effects (not reported). For instance, the GATE we estimate for men below age 50 with any pre-lottery primary care treatable ED use implies a 21 percentage point increase in the likelihood of using the emergency department upon gaining coverage. The forests do not reveal statistically significant group effects for other pre-lottery ED utilization or lottery list variables.

Figure 4 contrasts the distributions for the four main groups identified by the causal forest in more detail. The graphs highlight the differences in the empirical effect distribution that translate into the average group effects. Panel (a) shows a noticeable difference in the empirical distribution of the individualized treatment effects by pre-lottery SNAP receipt. The distribution of the ITEs for SNAP recipients is narrower and exhibits a denser mass in the positive effects region. This difference in the distribution of the heterogeneous effects reflects in the aggregated group average effect. Previous SNAP recipients are estimated to increase ED use by a statistically significant 9 
percentage points, while there is no effect for SNAP non-recipients.

Interestingly, panel (b) of Figure 4 shows that the ITE distribution by pre-lottery primary care treatable ED use exhibits a similar pattern to prior SNAP receipt, but with a bigger group effect for those with any prior visit of 13 percentage points. Finally, Panels (c) and (d) display the heterogeneous effects for age group (age $<50$ vs. age $\geq 50$ years) and gender (male vs. female). In contrast to the previous groups, the distributions for partitioning these groups are similar in shape but shifted to the right for the younger age group and men.

Second, the only subgroups with sizable negative effects are older adults (aged 50 and above) and those with any pre-lottery emergent, preventable ED visits. The effect for older adults is a 6percentage-point reduction in the probability of using the ED. However, the estimate is insignificant ( $p$-value $=0.24)$, possibly due to a lack of power. Further analyses in this direction suggest that those who reduce inpatient visits are mostly older people-individuals in the left tail of Figures 1c and 2a are older.

Finally, we divide the sample based on the individualized treatment effects to contrast the characteristics of those who increase and decrease usage ex-post. Table 5 presents the mean characteristics of individuals identified to increase and reduce emergency department utilization on the extensive margin. ${ }^{11}$ In almost all cases, we find that those who increase and decrease are different on observed dimensions. To highlight a few key differences, those estimated to increase the likelihood of any ED use are almost three years younger (39.8 vs. 41.5 years), less likely to be female (0.51 vs. 0.63$)$, more likely to have previously received SNAP (0.62 vs. 0.35 ) with higher total household benefits averaging ( $\$ 1,607$ vs. $\$ 717$ ), and more likely to have been a previous TANF recipient (0.03 vs. 0.01) with higher benefits (\$111 vs. \$63). In terms of prior ED visits, those estimated to increase use have a higher number of visits across all visits. The largest mean differences are for the overall number of ED visits and outpatient ED visits.

${ }^{11}$ The corresponding summary of characteristics for the intensive margin of ED use are available in Appendix A. 
Table 4: Group average treatment effects of Medicaid on the propensity of ED use

\begin{tabular}{|c|c|c|c|c|}
\hline \multirow[b]{2}{*}{ Group } & \multicolumn{3}{|c|}{ GRF estimates } & \multirow[b]{2}{*}{$\% \mathrm{~N}$} \\
\hline & GATE & SE & $p$-value & \\
\hline Female: & 0.00 & 0.04 & 0.97 & 0.55 \\
\hline Male: & 0.10 & 0.04 & 0.01 & 0.45 \\
\hline Gave phone number: No & 0.00 & 0.08 & 0.99 & 0.13 \\
\hline Gave phone number: Yes & 0.05 & 0.03 & 0.11 & 0.87 \\
\hline English as preferred language: No & 0.01 & 0.07 & 0.88 & 0.14 \\
\hline English as preferred language: Yes & 0.05 & 0.03 & 0.12 & 0.86 \\
\hline First week sign-up: No & 0.04 & 0.04 & 0.31 & 0.62 \\
\hline First week sign-up: Yes & 0.06 & 0.04 & 0.20 & 0.38 \\
\hline Pre-lottery SNAP recipient: No & -0.01 & 0.05 & 0.79 & 0.46 \\
\hline Pre-lottery SNAP recipient: Yes & 0.09 & 0.03 & 0.01 & 0.54 \\
\hline Pre-lottery TANF recipient: No & 0.04 & 0.03 & 0.17 & 0.98 \\
\hline Pre-lottery TANF recipient: Yes & 0.05 & 0.18 & 0.79 & 0.02 \\
\hline Age $\geq 50:$ No & 0.08 & 0.03 & 0.02 & 0.75 \\
\hline Age $\geq 50:$ Yes & -0.06 & 0.05 & 0.24 & 0.25 \\
\hline Two+ household members on lottery list: No & 0.03 & 0.03 & 0.42 & 0.80 \\
\hline Two+ household members on lottery list: Yes & 0.09 & 0.06 & 0.15 & 0.20 \\
\hline Any pre-lottery ED visit No & 0.03 & 0.03 & 0.34 & 0.69 \\
\hline Any pre-lottery ED visit: Yes & 0.06 & 0.05 & 0.24 & 0.31 \\
\hline Any pre-lottery on-hours ED visit: No & 0.03 & 0.03 & 0.35 & 0.77 \\
\hline Any pre-lottery on-hours ED visit: Yes & 0.07 & 0.05 & 0.18 & 0.23 \\
\hline Any pre-lottery off-hours ED visit: No & 0.04 & 0.03 & 0.21 & 0.81 \\
\hline Any pre-lottery off-hours ED visit: Yes & 0.06 & 0.06 & 0.29 & 0.19 \\
\hline Any pre-lottery emergent, non-preventable ED visit: No & 0.04 & 0.03 & 0.19 & 0.87 \\
\hline Any pre-lottery emergent, non-preventable ED visit: Yes & 0.06 & 0.07 & 0.40 & 0.13 \\
\hline Any pre-lottery emergent, preventable ED visit: No & 0.05 & 0.03 & 0.10 & 0.92 \\
\hline Any pre-lottery emergent, preventable ED visit: Yes & -0.05 & 0.08 & 0.55 & 0.08 \\
\hline Any pre-lottery primary care treatable ED visit: No & 0.02 & 0.03 & 0.59 & 0.81 \\
\hline Any pre-lottery primary care treatable ED visit: Yes & 0.13 & 0.06 & 0.03 & 0.19 \\
\hline Any pre-lottery non-emergent ED visit: No & 0.03 & 0.03 & 0.34 & 0.86 \\
\hline Any pre-lottery non-emergent ED visit: Yes & 0.08 & 0.07 & 0.21 & 0.14 \\
\hline
\end{tabular}

Notes: This table reports the group average treatment effects of Medicaid based on generalized random forests. The baseline sample consists of 24,615 individuals in the Taubman et al. (2014) sample with non-missing information on pre-lottery emergency department utilization and SNAP/TANF receipt. 
Figure 4: Distribution of individualized treatment effects of Medicaid by selected groups (Any visit)
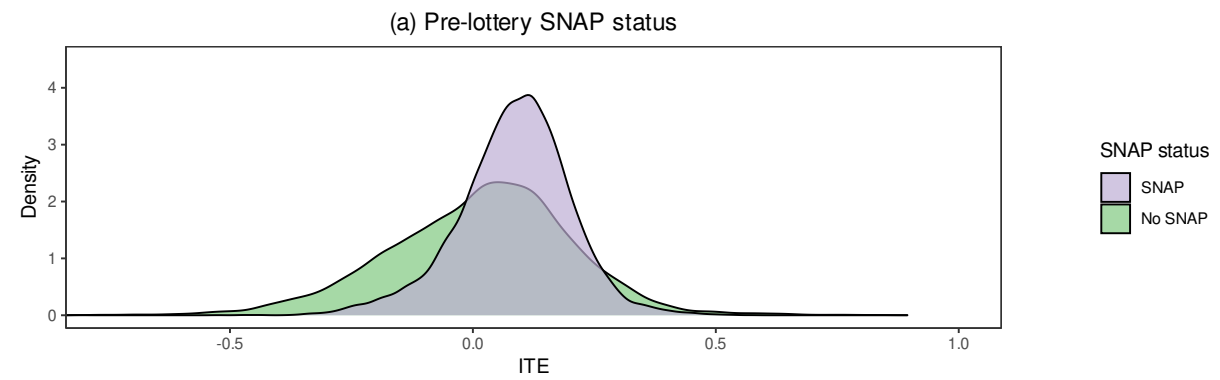

(b) Pre-lottery primary care treatable ED use

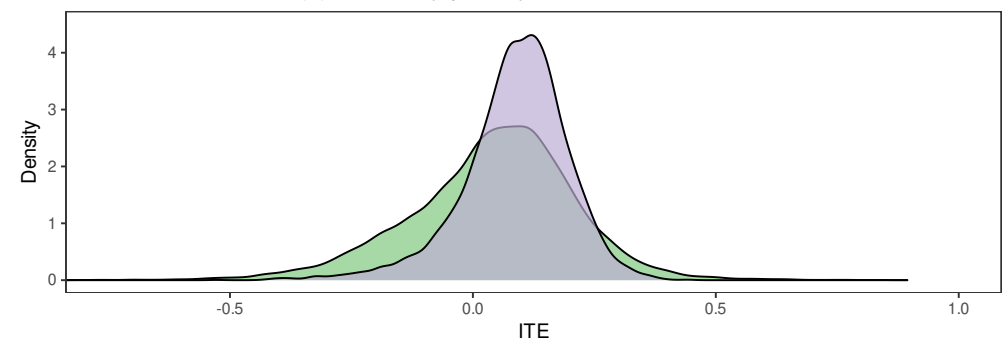

Pre-lottery p.c. treat. ED use

Any prior p.c. treat. ED use

No prior p.c. treat. ED use

(c) Age group

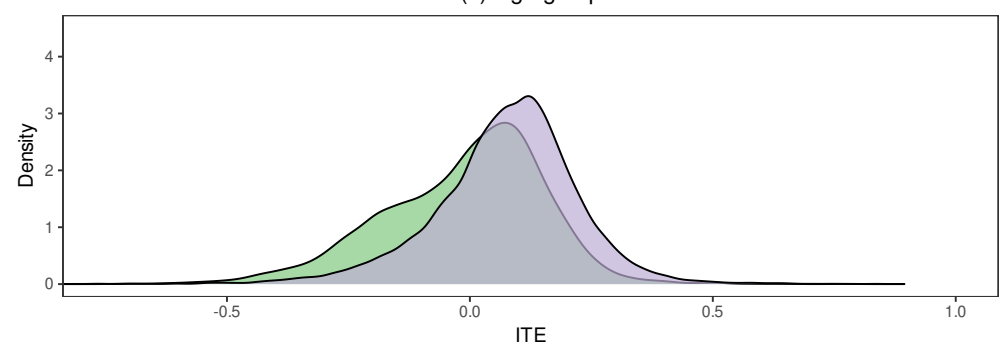

Age group

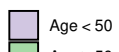

(d) Gender

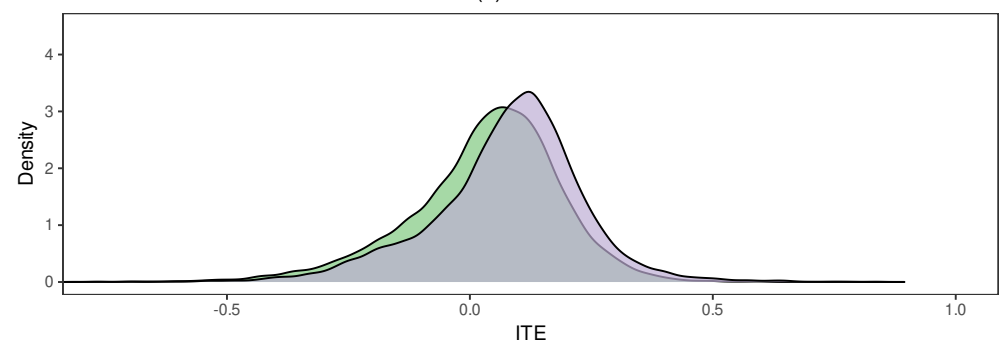

Notes: This figure plots the individualized treatment effects of Medicaid on any overall ED visit for the four major groups identified with substantial group average effects_-pre-lottery SNAP receipt (panel a), pre-lottery primary care treatable ED visit (panel b), age group (panel c), and gender (panel d). The baseline sample consists of 24,615 individuals in the Taubman et al. (2014) sample with non-missing information on pre-lottery emergency department utilization and SNAP/TANF receipt. 
Table 5: Characteristics of individuals who increased and decreased ED use

\begin{tabular}{|c|c|c|c|}
\hline Variable & $\begin{array}{l}\text { Increased } \\
\text { ED use } \\
\text { (1) }\end{array}$ & $\begin{array}{l}\text { Decreased } \\
\text { ED use } \\
\text { (2) }\end{array}$ & $\begin{array}{l}\text { Difference } \\
\text { (2)-(1) }\end{array}$ \\
\hline \multicolumn{4}{|l|}{ Lottery list characteristics } \\
\hline Age (years) & 38.76 & 41.48 & $-2.72 * * *$ \\
\hline Gave phone number & 0.87 & 0.87 & 0.00 \\
\hline English as preferred language & 0.87 & 0.85 & $0.02 * * *$ \\
\hline Female & 0.51 & 0.63 & $-0.12 * * *$ \\
\hline Week of lottery sign up & 1.57 & 1.61 & -0.04 \\
\hline Provided P.O. box address & 0.02 & 0.03 & $-0.01 * * *$ \\
\hline Signed up self for lottery & 0.89 & 0.92 & $-0.03 * * *$ \\
\hline Pre-lottery SNAP recipient & 0.62 & 0.35 & $0.27 * * *$ \\
\hline Pre-lottery SNAP benefit amount (\$) & 1607.33 & 713.38 & $893.95 * * *$ \\
\hline Pre-lottery TANF recipient & 0.03 & 0.01 & $0.02 * * *$ \\
\hline Pre-lottery TANF benefit amount (\$) & 111.08 & 63.09 & $47.99 * * *$ \\
\hline \multicolumn{4}{|l|}{ Pre-lottery ED usage } \\
\hline Number of overall visits & 0.88 & 0.49 & $0.39 * * *$ \\
\hline Number of inpatient visits & 0.09 & 0.07 & $0.02 * * *$ \\
\hline Number of outpatient visits & 0.78 & 0.42 & $0.36 * * *$ \\
\hline Number of on-hours visits & 0.51 & 0.30 & $0.21 * * *$ \\
\hline Number of off-hours visits & 0.37 & 0.20 & $0.17 * * *$ \\
\hline Number of emergent, non-preventable visits & 0.18 & 0.09 & $0.09 * * *$ \\
\hline Number of emergent, preventable visits & 0.07 & 0.04 & $0.03 * * *$ \\
\hline Number of primary care treatable visits & 0.30 & 0.17 & $0.13 * * *$ \\
\hline Number of non-emergent visits & 0.18 & 0.10 & $0.08 * * *$ \\
\hline Number ambulatory-care-sensitive visits & 0.05 & 0.04 & $0.01 * * *$ \\
\hline Number of visits (chronic conditions) & 0.14 & 0.11 & $0.03 * * *$ \\
\hline Number of visits (injury) & 0.20 & 0.09 & $0.11 * * *$ \\
\hline Number of visits (skin conditions) & 0.05 & 0.03 & $0.02 * * *$ \\
\hline Number of visits (abdominal pain) & 0.04 & 0.02 & $0.02 * * *$ \\
\hline Number of visits (back pain) & 0.04 & 0.02 & $0.02 * * *$ \\
\hline Number of visits (chest pain) & 0.02 & 0.02 & $0.00 * * *$ \\
\hline Number of visits (headache) & 0.03 & 0.02 & $0.01 * * *$ \\
\hline Number of visits (mood disorders) & 0.02 & 0.02 & 0.00 \\
\hline Number of visits (psychiatric conditions) & 0.06 & 0.05 & $0.01 *$ \\
\hline Sum of total ED charges & 1013.28 & 584.41 & $428.87 * * *$ \\
\hline $\mathrm{N}$ & 16816 & 7797 & 24613 \\
\hline
\end{tabular}

Notes: This table reports the means of individual characteristics and pre-randomization ED use for those estimated to increase and decrease ED use upon receiving Medicaid coverage based on the generalized random forest ITE estimates. The sample consists of 24,613 individuals in the Taubman et al. (2014) sample with non-missing information on pre-lottery emergency department utilization and SNAP/TANF receipt. 


\subsection{Results of policy learning exercises}

This section presents the results of the policy learning exercises described in Section 3.2 using the estimated heterogeneous effects. Until now, we have focused on the effects of Medicaid coverage (i.e., the conditional LATE). However, the policy learning exercises are based on the intent-to-treat effects of winning the lottery reported in Appendix B. The heterogeneous intent-to-treat effects follow a similar pattern as the LATE coverage effects of Medicaid but the former is more natural for the policy learning exercises because it is the lottery and not the actual coverage that is random.

\subsubsection{Comparing the observed lottery to an alternative assignment scheme}

In this theoretical exercise, our objective is to estimate an alternative allocation scheme that prioritizes those least likely to increase unnecessary ED use, defined as the number of non-emergent ED visits. Doing so allows us to describe how the sample selected by such an optimal allocation scheme would compare to the sample of observed lottery winners. Again, while this exercise is infeasible from the point of policy-making, we use it to illustrate the value of the heterogeneous effects and verify that, given a well-defined objective, a random assignment scheme may be inferior to one based on the empirical distribution of treatment effects.

Following Kitagawa \& Tetenov (2018), we first compute the optimal treatment allocation scheme that minimizes the objective function of the number of non-emergent ED utilization using integer programming. We constrain the number of people selected by the allocation scheme to be the same number that won the Oregon lottery. We then compare the selected sample to the original lottery winners. As expected in Table 6, the selected sample based on the optimal allocation scheme has substantially lower ex-post ED utilization for non-emergent causes (0.18 fewer visits). Other ED use measures following treatment are also substantially lower. The same finding holds for pre-lottery ED use, suggesting that ED use patterns are complex and treatment effects are driven by a population that uses the ED for most of their health care needs (urgent or non-urgent). Comparing the magnitude of pre-and post-randomization ED use outcomes within the same category suggests larger differences between the two samples, indicating that the estimated optimal assignment scheme 


\section{Table 6: Individuals selected by lottery and the optimal linear program}

\begin{tabular}{|c|c|c|c|}
\hline Variable & $\begin{array}{c}\text { Selected by lottery } \\
\text { (1) }\end{array}$ & $\begin{array}{l}\text { Selected by optimal } \\
\text { linear program } \\
\text { (2) }\end{array}$ & $\begin{array}{l}\text { Difference } \\
\text { (2)-(1) }\end{array}$ \\
\hline \multicolumn{4}{|l|}{ Lottery list and baseline characteristics } \\
\hline Age (years) & 39.50 & 40.16 & $0.66 * * *$ \\
\hline Gave phone number & 0.88 & 0.87 & -0.01 \\
\hline English as preferred language & 0.85 & 0.79 & $-0.06 * * *$ \\
\hline Female & 0.54 & 0.53 & -0.01 \\
\hline Week of lottery sign up & 1.55 & 1.66 & $0.11 * * *$ \\
\hline Provided P.O. box address & 0.03 & 0.03 & 0.00 \\
\hline Signed up self for lottery & 0.85 & 0.85 & 0.00 \\
\hline Pre-lottery SNAP recipient & 0.53 & 0.43 & $-0.10 * * *$ \\
\hline Pre-lottery SNAP benefit amount (\$) & 1376.53 & 1120.72 & $-255.81 * * *$ \\
\hline Pre-lottery TANF recipient & 0.02 & 0.02 & $0.00 * * *$ \\
\hline Pre-lottery TANF benefit amount $(\$)$ & 100.09 & 72.82 & $-27.27 * * *$ \\
\hline Number of overall visits & 0.71 & 0.44 & $-0.27 * * *$ \\
\hline \multicolumn{4}{|l|}{ Pre-randomization ED use } \\
\hline Number of inpatient visits & 0.08 & 0.05 & $-0.03 * * *$ \\
\hline Number of outpatient visits & 0.63 & 0.39 & $-0.24 * * *$ \\
\hline Number of on-hours visits & 0.41 & 0.25 & $-0.16^{* * *}$ \\
\hline Number of off-hours visits & 0.30 & 0.19 & $-0.11 * * *$ \\
\hline Number of emergent, non-preventable visits & 0.15 & 0.09 & $-0.06^{* * *}$ \\
\hline Number of emergent, preventable visits & 0.05 & 0.03 & $-0.02 * * *$ \\
\hline Number of primary care treatable visits & 0.25 & 0.15 & $-0.10 * * *$ \\
\hline Number of non-emergent visits & 0.14 & 0.08 & $-0.06 * * *$ \\
\hline Number ambulatory-care-sensitive visits & 0.05 & 0.03 & $-0.02 * * *$ \\
\hline Number of visits (chronic conditions) & 0.12 & 0.08 & $-0.04 * * *$ \\
\hline Number of visits (injury) & 0.17 & 0.10 & $-0.07 * * *$ \\
\hline Number of visits (skin conditions) & 0.04 & 0.03 & $-0.01 * * *$ \\
\hline Number of visits (abdominal pain) & 0.03 & 0.02 & $-0.01 * * *$ \\
\hline Number of visits (back pain) & 0.03 & 0.02 & $-0.01 * * *$ \\
\hline Number of visits (chest pain) & 0.02 & 0.01 & $-0.01 * * *$ \\
\hline Number of visits (headache) & 0.02 & 0.01 & $-0.01 * * *$ \\
\hline Number of visits (mood disorders) & 0.02 & 0.01 & $-0.01 * *$ \\
\hline Number of visits (psychiatric conditions) & 0.05 & 0.03 & $-0.02 * * *$ \\
\hline Sum of total ED charges & 808.36 & 504.40 & $-303.96 * * *$ \\
\hline Number of overall visits & 0.98 & 0.43 & $-0.55^{* * *}$ \\
\hline \multicolumn{4}{|l|}{ ED use outcomes } \\
\hline Number of inpatient visits & 0.10 & 0.06 & $-0.04 * * *$ \\
\hline Number of outpatient visits & 0.87 & 0.36 & $-0.51 * * *$ \\
\hline Number of emergent, non-preventable visits & 0.20 & 0.10 & $-0.10 * * *$ \\
\hline Number of emergent, preventable visits & 0.07 & 0.04 & $-0.03 * * *$ \\
\hline Number of primary care treatable visits & 0.35 & 0.17 & $-0.18 * * *$ \\
\hline Number of non-emergent visits & 0.20 & 0.02 & $-0.18 * * *$ \\
\hline $\mathrm{N}$ & 9607 & 9607 & \\
\hline
\end{tabular}

Notes: This table reports the means of individual characteristics, pre-randomization ED use and post-treatment outcomes for those selected by the lottery and selected by the optimal treatment assignment solution based on the optimal linear program which minimizes non-emergent ED use under the restriction that the same number of people are treated as within the lottery. The sample consists of 24,605 individuals in the Taubman et al. (2014) sample with non-missing information on pre-lottery emergency department utilization and SNAP/TANF receipt. 
successfully selects those who react to obtaining coverage by reducing ED usage. For instance, the difference in outpatient use between the two samples in the pre-lottery period is 0.24 visits, while the same difference in the post-lottery period is 0.51 visits.

\subsubsection{Identifying people at risk of non-emergent ED use via decision rules}

A more practical way to use the estimated heterogeneous effects for policy-making is to design simple decision rules identifying people at greater risk of increasing non-emergent ED use among Medicaid enrollees. Here, the administrator may still use a lottery to solicit Medicaid applications but target a subset of new enrollees with an outreach or educational intervention. For instance, policymakers may want to identify some enrollees who may be required to establish primary care upon receiving Medicaid coverage. Of course, a straightforward decision rule might be to target the intervention based on their predicted individualized treatment effects, such as those with $\widehat{\tau}(X) \geq 0$. However, Athey \& Imbens (2019) points out that such decision rules are not always optimal in the sense of minimizing the loss from not using the ideal assignment rule or policy.

Thus, we estimate the depth-2 decision trees to identify those most likely to increase nonemergent ED visits using only a subset of pre-lottery ED use variables (Athey \& Wager 2021). Figure 5 presents the estimated decision rule. The first node of the decision tree partitions the sample based on a threshold of two prior outpatient ED visits. On the one hand, for people with two or fewer outpatient ED visits, the decision rule selects them if they had no previous ED use classified as emergent. This decision rule is interesting because, given the objective of maximizing non-emergent use, it identifies those with no prior emergent use as those likely to increase nonemergent use.

On the other hand, for those with more than two previous outpatient ED visits (potentially excessive ED use), those with at most one prior ED visit resulting in hospitalization are selected. Again, this rule reasonably targets those who rarely used the ED for conditions severe enough to warrant inpatient care in the past.

We emphasize that our estimated decision rule is not set in stone and may be impractical, 


\section{Figure 5: Decision rule for targeting outreach based on non-emergent ED use}

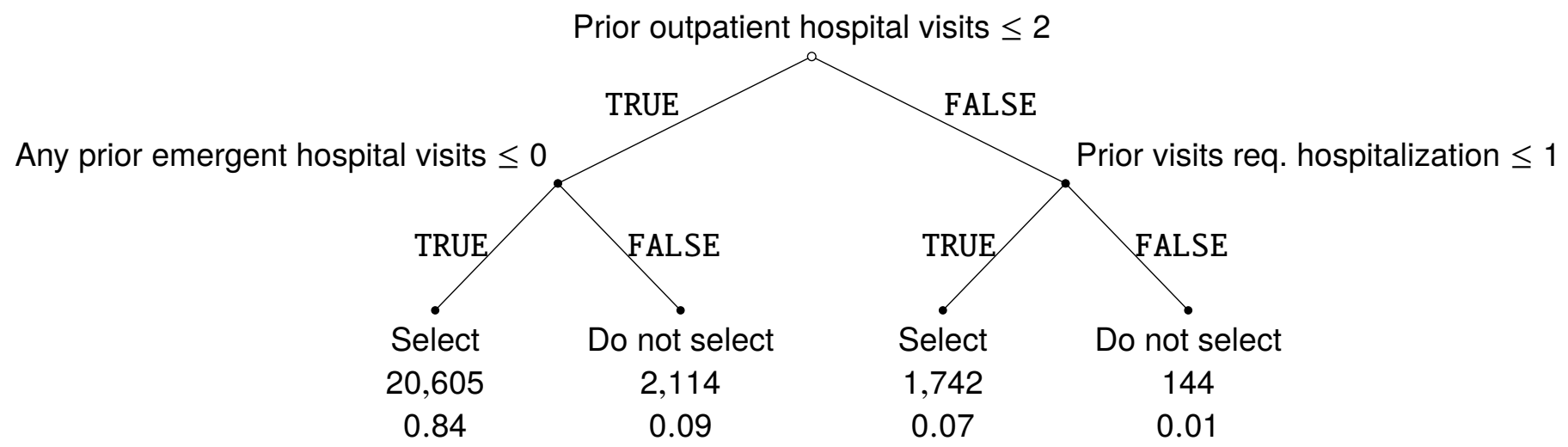

Notes: This figure shows the optimal policy assignment rule based on the doubly robust scores of the conditional average treatment effect (CATE) of being randomly selected as a lottery winner in the Oregon Health Insurance Experiment using the efficient policy learning framework of Athey \& Wager (2021). The sample consists of 24,615 individuals in the Taubman et al. (2014) sample with non-missing information on pre-lottery emergency department utilization and SNAP/TANF receipt.

especially if the previous ED utilization variables are not easily accessible. Policymakers could also specify a different objective function to align with their goals or accommodate additional constraints. Moreover, the set of variables included in the estimation of the decision rule can be modified to respect fairness or other political/legal requirements.

\section{Conclusion}

This paper estimates the heterogeneous impacts of Medicaid coverage on emergency department visits using records from 12 hospitals in Portland, Oregon, matched to the Oregon Health Insurance Experiment. The result that Medicaid increased ED utilization in the 2008 Oregon Health Experiment made headlines because of the hypothesis that insurance coverage should make it easier for recipients to access primary care and reduce the need to use the emergency department. However, this is an empirical question, given that insurance coverage also reduces out-of-pocket healthcare spending. We provide new insights into the ED results of the Oregon experiment when we go beyond the average impacts of Medicaid coverage. We do so by estimating the individualized treatment effects of Medicaid using nonparametric machine learning methods. We then leverage the heterogeneous effects to estimate decision rules to illustrate their usefulness for policymakers 
in similar settings.

We find substantial treatment effect heterogeneity in the impacts of Medicaid on emergency department utilization. The individualized treatment effects of Medicaid on ED use indicate positive and negative effects, suggesting a more nuanced interpretation of Medicaid's average impacts. We also find that coverage effects for different types of ED use exhibit meaningful heterogeneity. Due to the variation in the individualized effect distribution, the average treatment effect is not typical but is driven by sections of the sample in the right tail of the distribution. A small proportion of high-use individuals drive the positive (average) Medicaid effect. Despite the positive average effects, the predicted treatment effects for many individuals are negative.

Our findings suggest that the average effect sometimes masks countervailing forces. In some instances, although many people increase ED usage due to obtaining coverage, a sizable proportion of people either decrease usage or do not respond. We find that reductions in ED use are pronounced for inpatient visits and emergent, non-preventable conditions. The overall null effects for these outcomes hide substantial opposing effects of obtaining coverage.

We also find substantial treatment effect heterogeneity on the intensive margin. Overall, the results suggest that increased ED usage is likely driven by intensive margin effects rather than extensive margin effects. Individuals who already utilize the emergency department further increase their usage after receiving Medicaid.

We also characterize the groups of people mainly driving the positive average Medicaid effects in the right tail of the distribution of the heterogeneous effects. Those with positive heterogeneous effects are predominantly younger, more likely to be men, more likely to receive SNAP and TANF in the pre-lottery period, and more likely to have higher baseline ED use. In particular, we identify four groups estimated to have statistically significant increases in ED use that are at least twice as large as the magnitude of the average effect. These groups are men, prior SNAP participants, younger adults under 50 years old, and people with pre-lottery ED use classified as primary care treatable.

Finally, we illustrate one way to use the estimated heterogeneous effects to estimate decision 
rules that may help to identify and target enrollees likely to increase non-emergent emergency department utilization. For people with minimal previous outpatient ED use, the decision rule identifies those with no previous emergent use as likely to increase non-emergent ED use. For those with higher previous outpatient ED use, the decision rule selects those whose ED visit resulted in hospitalization only once (i.e., those who have rarely presented with severe health problems justifying inpatient treatment). While our decision rules are illustrative, they highlight the potential for using statistical decision rules to guide policymakers to achieve context-specific objectives. 


\section{References}

Abadie, A. (2003), 'Semiparametric instrumental variable estimation of treatment response models', Journal of econometrics 113(2), 231-263.

Adams, J. G. (2013), 'Emergency department overuse: perceptions and solutions', Jama 309(11), 1173-1174.

Allen, H., Baicker, K., Finkelstein, A., Taubman, S., Wright, B. J. \& Group, O. H. S. (2010), 'What the oregon health study can tell us about expanding medicaid', Health Affairs 29(8), 1498-1506.

Allen, H., Baicker, K., Taubman, S., Wright, B. \& Finkelstein, A. (2013), 'The oregon health insurance experiment: when limited policy resources provide research opportunities', Journal of health politics, policy and law 38(6), 1183-1192.

Angrist, J. D. (2004), 'Treatment effect heterogeneity in theory and practice', The economic journal 114(494), C52-C83.

Angrist, J. D., Imbens, G. W. \& Rubin, D. B. (1996), 'Identification of causal effects using instrumental variables', Journal of the American statistical Association 91(434), 444-455.

Athey, S. \& Imbens, G. W. (2019), 'Machine learning methods that economists should know about', Annual Review of Economics 11, 685-725.

Athey, S., Tibshirani, J., Wager, S. et al. (2019), 'Generalized random forests', The Annals of Statistics 47(2), 1148-1178.

Athey, S. \& Wager, S. (2021), 'Policy learning with observational data', Econometrica 89(1), 133161.

Baicker, K., Taubman, S. L., Allen, H. L., Bernstein, M., Gruber, J. H., Newhouse, J. P., Schneider, E. C., Wright, B. J., Zaslavsky, A. M. \& Finkelstein, A. N. (2013), 'The oregon experiment-effects of medicaid on clinical outcomes', New England Journal of Medicine 368(18), 1713-1722.

Billings, J., Parikh, N. \& Mijanovich, T. (2000), 'Emergency room use: the new york story', Issue Brief (Commonw Fund) 434, 1-12.

Breiman, L. (2001), 'Random forests', Machine learning 45, 5-32.

Chen, C., Scheffler, G. \& Chandra, A. (2011), 'Perspective: Massachusetts' health care reform and emergency department utilization', $N$ Engl J Med. 365, e25.

Chernozhukov, V., Chetverikov, D., Demirer, M., Duflo, E., Hansen, C., Newey, W. K. \& Robins, J. (2018), 'Double/debiased machine learning for treatment and structural parameters', The Econometrics Journal 21(1), C1-C68.

Chou, S.-C., Gondi, S., Weiner, S. G., Schuur, J. D. \& Sommers, B. D. (2020), 'Medicaid expansion reduced emergency department visits by low-income adults due to barriers to outpatient care', Medical Care 58(6), 511-518. 
Deaton, A. (2010), 'Instruments, randomization, and learning about development', Journal of economic literature 48(2), 424-55.

Dehejia, R. H. (2005), 'Program evaluation as a decision problem', Journal of Econometrics 125(12), 141-173.

Finkelstein, A. N., Taubman, S. L., Allen, H. L., Wright, B. J. \& Baicker, K. (2016), 'Effect of medicaid coverage on ed use-further evidence from oregon's experiment', $N$ Engl J Med 375(16), 1505-1507.

Finkelstein, A., Taubman, S., Wright, B., Bernstein, M., Gruber, J., Newhouse, J. P., Allen, H., Baicker, K. \& Group, O. H. S. (2012), 'The oregon health insurance experiment: evidence from the first year', The Quarterly journal of economics 127(3), 1057-1106.

Garthwaite, C., Graves, J. A., Gross, T., Karaca, Z., Marone, V. R. \& Notowidigdo, M. J. (2019), All medicaid expansions are not created equal: the geography and targeting of the affordable care act, Technical report, National Bureau of Economic Research.

Giannouchos, T. V., Ukert, B. \& Andrews, C. (2022), 'Association of medicaid expansion with emergency department visits by medical urgency', JAMA Network Open 5(6), e2216913-e2216913.

Hastie, T., Tibshirani, R., Friedman, J. H. \& Friedman, J. H. (2009), The elements of statistical learning: data mining, inference, and prediction, Vol. 2, Springer.

Heckman, J. J., Smith, J. \& Clements, N. (1997), 'Making the most out of programme evaluations and social experiments: Accounting for heterogeneity in programme impacts', The Review of Economic Studies 64(4), 487-535.

Hirano, K. \& Porter, J. R. (2009), 'Asymptotics for statistical treatment rules', Econometrica 77(5), 1683-1701.

Imbens, G. W., Angrist, J. D. et al. (1994), 'Identification and estimation of local average treatment effects', Econometrica 62(2), 467-475.

Kitagawa, T. \& Tetenov, A. (2018), 'Who should be treated? empirical welfare maximization methods for treatment choice', Econometrica 86(2), 591-616.

Knaus, M. C., Lechner, M. \& Strittmatter, A. (2021), 'Machine learning estimation of heterogeneous causal effects: Empirical monte carlo evidence', The Econometrics Journal 24(1), 134-161.

Kowalski, A. E. (2021), 'Reconciling seemingly contradictory results from the oregon health insurance experiment and the massachusetts health reform', Review of Economics and Statistics pp. $1-45$.

Lechner, M., Strittmatter, A. \& Knaus, M. (2020), 'Heterogeneous employment effects of job search programmes: A machine learning approach', Journal of Human Resources .

List, J. A., Shaikh, A. M. \& Xu, Y. (2019), 'Multiple hypothesis testing in experimental economics', Experimental Economics 22(4), 773-793. 
Manski, C. F. (2004), 'Statistical treatment rules for heterogeneous populations', Econometrica 72(4), 1221-1246.

Marx, P. (2020), 'Sharp bounds in the latent index selection model', arXiv preprint arXiv:2012.02390 .

Miller, S. (2012), 'The effect of insurance on emergency room visits: an analysis of the 2006 massachusetts health reform', Journal of public Economics 96(11-12), 893-908.

Moore, B. J. \& Liang, L. (2020), Costs of emergency department visits in the united states, 2017, in 'Healthcare Cost and Utilization Project (HCUP) Statistical Briefs [Internet]', Agency for Healthcare Research and Quality (US).

Sabbatini, A. K. \& Dugan, J. (2022), 'Medicaid expansion and avoidable emergency department use-implications for us national and state government spending', JAMA Network Open 5(6), e2216917-e2216917.

Sexton, J. \& Laake, P. (2009), 'Standard errors for bagged and random forest estimators', Computational Statistics \& Data Analysis 53(3), 801-811.

Słoczyński, T. (2020), 'When should we (not) interpret linear iv estimands as late?', arXiv preprint arXiv:2011.06695 .

Sommers, B. D., Blendon, R. J., Orav, E. J. \& Epstein, A. M. (2016), 'Changes in utilization and health among low-income adults after medicaid expansion or expanded private insurance', JAMA internal medicine 176(10), 1501-1509.

Sommers, B. D. \& Simon, K. (2017), 'Health insurance and emergency department use-a complex relationship', The New England journal of medicine 376(18), 1708-1711.

Stoye, J. (2009), 'Minimax regret treatment choice with finite samples', Journal of Econometrics 151(1), 70-81.

Stoye, J. (2012), 'Minimax regret treatment choice with covariates or with limited validity of experiments', Journal of Econometrics 166(1), 138-156.

Tang, N., Stein, J., Hsia, R. Y., Maselli, J. H. \& Gonzales, R. (2010), 'Trends and characteristics of us emergency department visits, 1997-2007', Jama 304(6), 664-670.

Taubman, S. L., Allen, H. L., Wright, B. J., Baicker, K. \& Finkelstein, A. N. (2014), 'Medicaid increases emergency-department use: evidence from oregon's health insurance experiment', Science 343(6168), 263-268.

Wager, S. \& Athey, S. (2018), 'Estimation and inference of heterogeneous treatment effects using random forests', Journal of the American Statistical Association 113(523), 1228-1242.

Woodworth, L. (2020), 'Swamped: Emergency department crowding and patient mortality', Journal of health economics 70, 102279. 
Young, A. (2019), 'Channeling fisher: Randomization tests and the statistical insignificance of seemingly significant experimental results*', The Quarterly Journal of Economics 134(2), 557-598.

Zhao, Y., Zeng, D., Rush, A. J. \& Kosorok, M. R. (2012), 'Estimating individualized treatment rules using outcome weighted learning', Journal of the American Statistical Association 107(499), 1106-1118. 


\section{A. Supplementary Material for Medicaid Coverage Effects}

\section{Figure A.1: Distribution of individualized treatment effects of Medicaid on the number of ED visits}

(a) Any visit

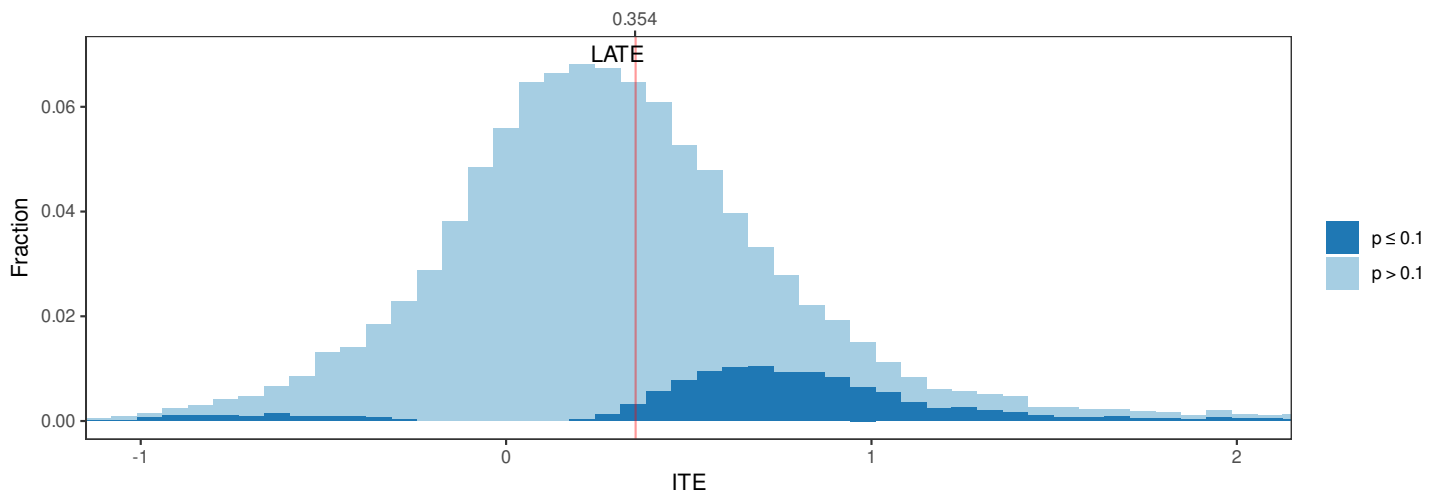

(b) Outpatient

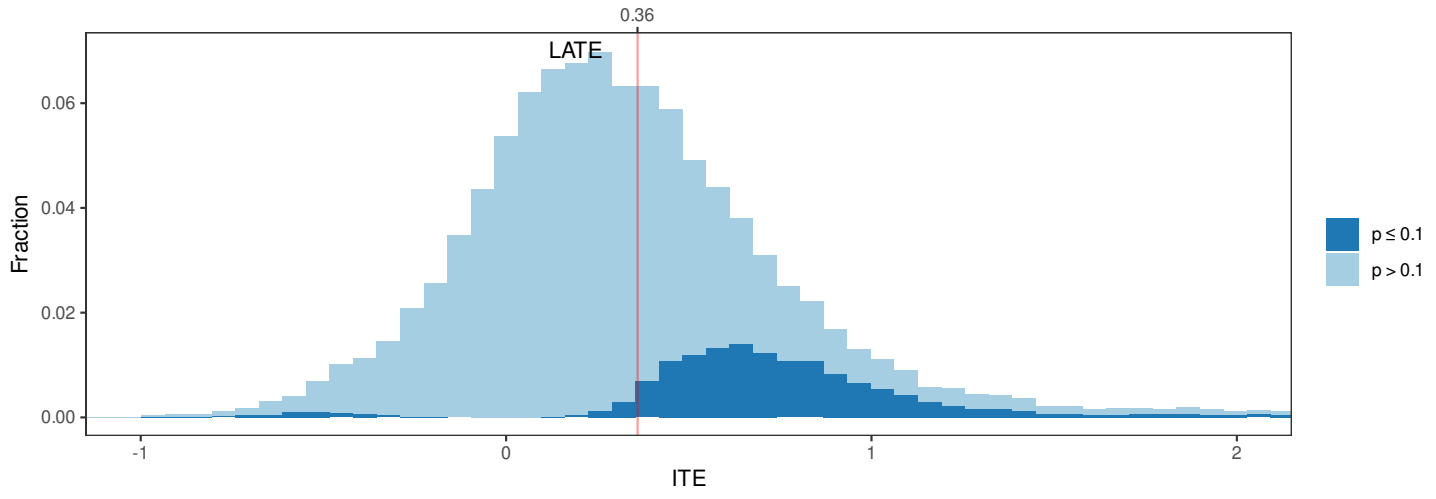

(c) Inpatient

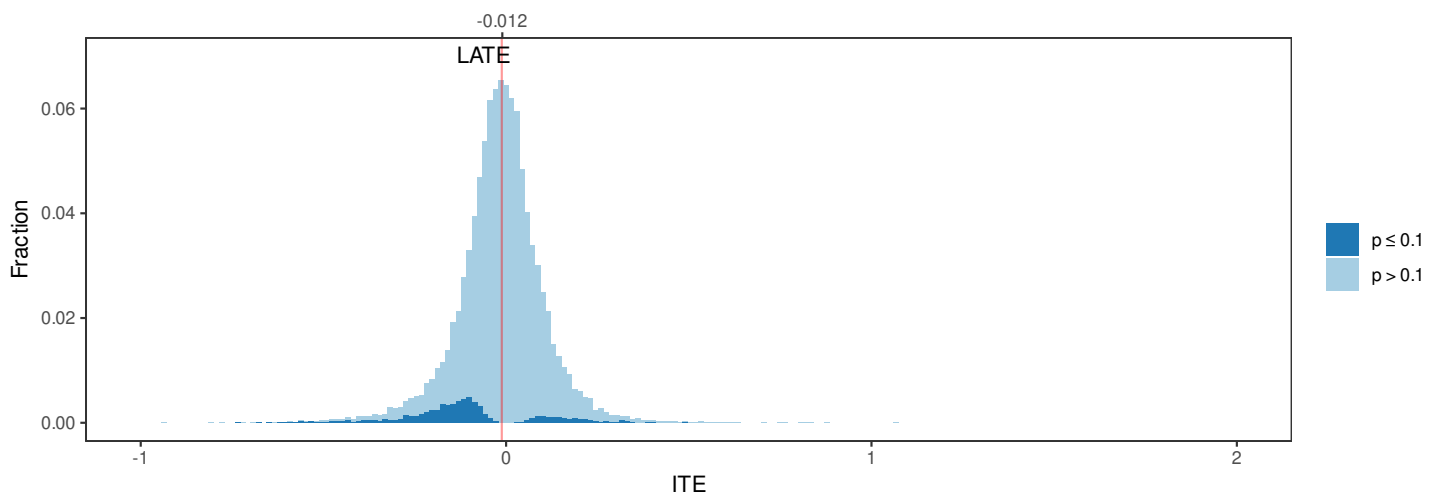

Notes: This figure plots the individualized treatment effects of Medicaid on the number of overall ED visit (panel a), the number of outpatient ED visits (panel b), and the number of inpatient ED use (panel c) based on generalized random forests. The darker shade denotes statistical significance at the $10 \%$ level. The red vertical line indicates the local average treatment effect. The baseline sample consists of 24,615 individuals in the Taubman et al. (2014) sample with non-missing information on pre-lottery emergency department utilization and SNAP/TANF receipt. The estimates displayed exclude less than half a percentile at the top and bottom of the distribution, resulting in the axes corresponding approximately to the percentile range $[0.5 \%, 99.5 \%]$. Bin size is chosen according to the Freedman-Diaconis rule. 


\section{Figure A.2: Distribution of individualized treatment effects of Medicaid on the number of ED visits by type of condition}

(a) Emergent, non-preventable

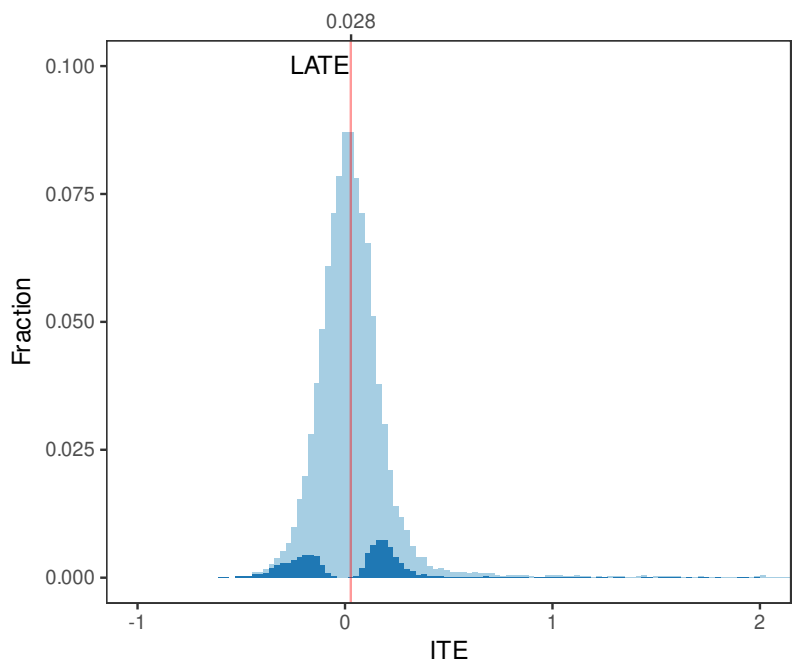

(c) Primary care treatable

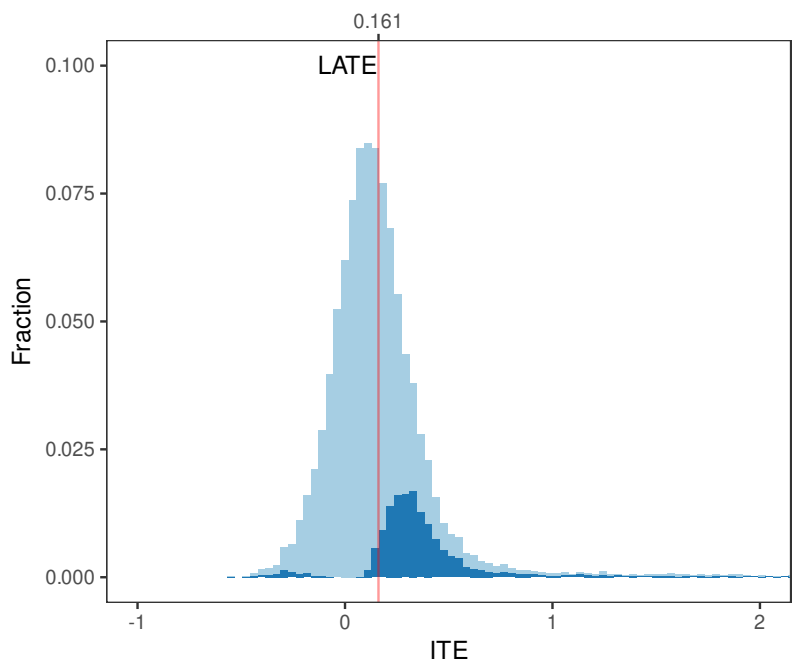

(b) Emergent, preventable

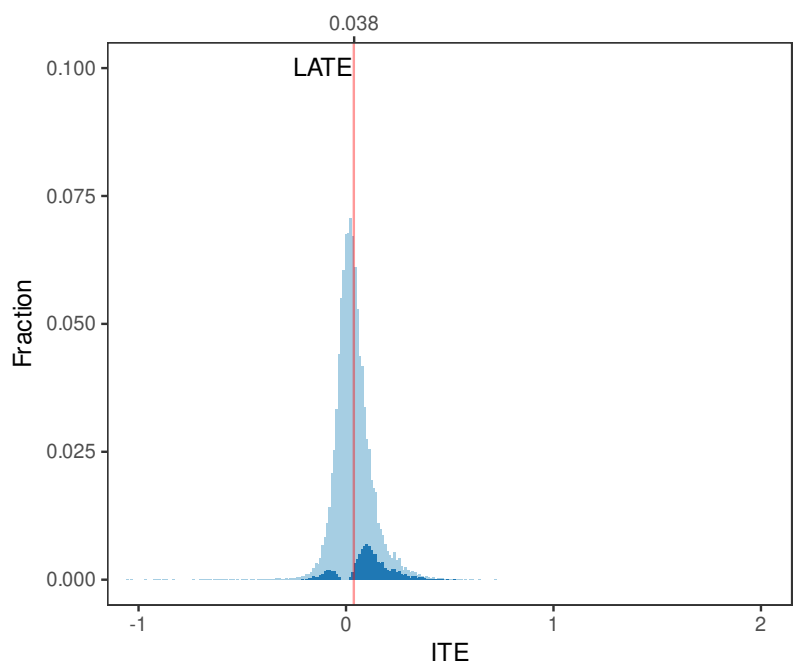

(d) Non-emergent

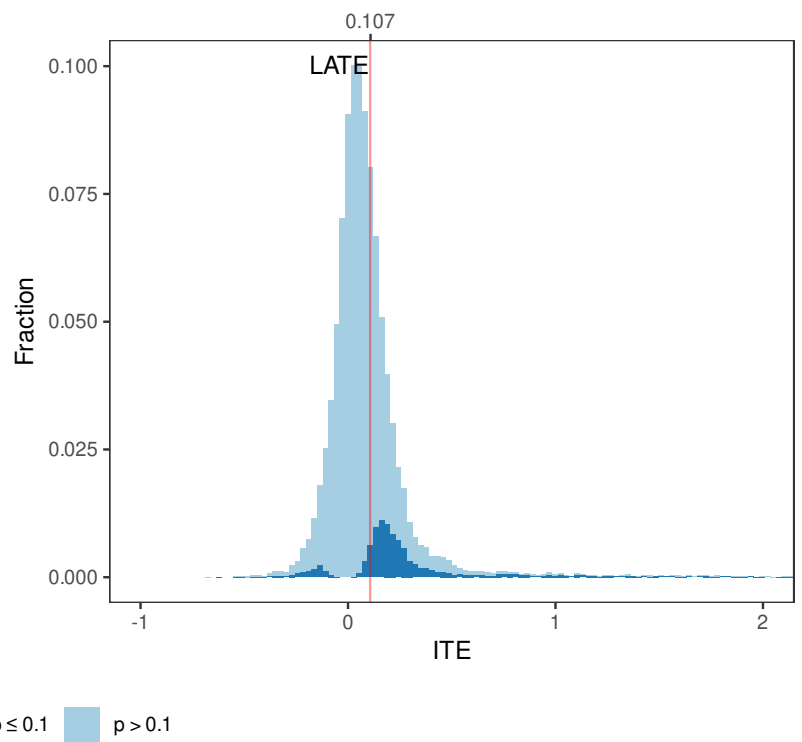

Notes: This figure plots the individualized treatment effects of Medicaid by type of ED visit based on generalized random forests for the number of emergent, non-preventable visits (panel a), the number of emergent, preventable visits (panel b), the number of primary care treatable visits (panel c), and the number of non-emergent visits (panel d). Measures of the type of ED visit are based on Billings et al.'s (2000) algorithm described in Taubman et al. (2014). The number of visits of each type are then obtained by summing the probabilities across all visits for an individual. The darker shade denotes statistical significance at the $10 \%$ level. The red vertical line indicates the local average treatment effect. The baseline sample consists of 24,615 individuals in the Taubman et al. (2014) sample with non-missing information on pre-lottery emergency department utilization and SNAP/TANF receipt. The estimates displayed exclude less than half a percentile at the top and bottom of the distribution, resulting in the axes corresponding approximately to the percentile range $[0.5 \%, 99.5 \%]$. Bin size is chosen according to the Freedman-Diaconis rule. 
Figure A.3: Variable importance scores in growing causal forest (Number of visits)

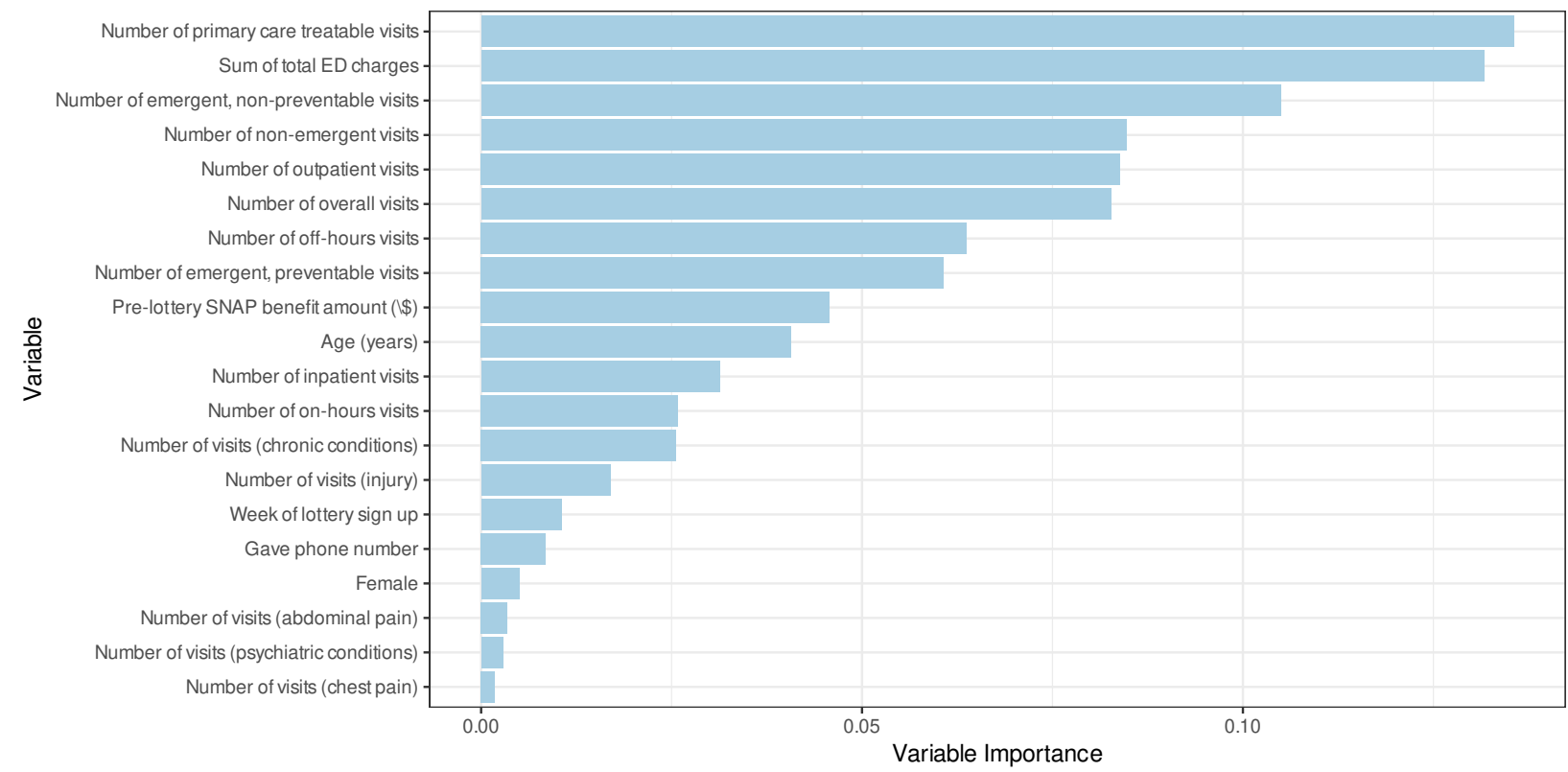

Notes This figure shows the variable importance scores of the top 20 characteristics in growing the generalized random forests used to estimate the individualized treatment effects of Medicaid for the number of overall ED visit. The variable importance measure is a simple weighted sum of the proportion of times a variable is used in a tree splitting step at each depth in growing the forest. The scores roughly capture how important a variable is for driving treatment effect heterogeneity. The baseline sample consists of 24,615 individuals in the Taubman et al. (2014) sample with non-missing information on pre-lottery emergency department utilization and SNAP/TANF receipt. 
Table A.1: Variable importance for all variables in growing causal forest (overall ED use)

\begin{tabular}{|c|c|c|c|}
\hline \multicolumn{2}{|l|}{ Any visit } & \multicolumn{2}{|l|}{ Number of visits } \\
\hline Variable & Importance & Variable & Importance \\
\hline Pre-lottery SNAP benefit amount (\$) & 0.18 & Number of primary care treatable visits & 0.14 \\
\hline Age (years) & 0.18 & Sum of total ED charges & 0.13 \\
\hline Sum of total ED charges & 0.09 & Number of emergent, non-preventable visits & 0.10 \\
\hline Number of emergent, non-preventable visits & 0.09 & Number of non-emergent visits & 0.08 \\
\hline Number of primary care treatable visits & 0.08 & Number of outpatient visits & 0.08 \\
\hline Week of lottery sign up & 0.05 & Number of overall visits & 0.08 \\
\hline Number of non-emergent visits & 0.05 & Number of off-hours visits & 0.06 \\
\hline Female & 0.04 & Number of emergent, preventable visits & 0.06 \\
\hline Number of emergent, preventable visits & 0.02 & Pre-lottery SNAP benefit amount (\$) & 0.05 \\
\hline Number of visits (chronic conditions) & 0.01 & Age (years) & 0.04 \\
\hline Number of inpatient visits & 0.01 & Number of inpatient visits & 0.03 \\
\hline Pre-lottery SNAP recipient & 0.01 & Number of on-hours visits & 0.03 \\
\hline Number of outpatient visits & 0.01 & Number of visits (chronic conditions) & 0.03 \\
\hline Number of off-hours visits & 0.01 & Number of visits (injury) & 0.02 \\
\hline Number of on-hours visits & 0.01 & Week of lottery sign up & 0.01 \\
\hline Number of overall visits & 0.01 & Gave phone number & 0.01 \\
\hline Signed up self for lottery & 0.01 & Female & 0.01 \\
\hline Gave phone number & 0.01 & Number of visits (abdominal pain) & 0.00 \\
\hline Number of visits (injury) & 0.01 & Number of visits (psychiatric conditions) & 0.00 \\
\hline English as preferred language & 0.00 & Number of visits (chest pain) & 0.00 \\
\hline Pre-lottery TANF benefit amount (\$) & 0.00 & Pre-lottery TANF benefit amount (\$) & 0.00 \\
\hline Number of visits (psychiatric conditions) & 0.00 & Number of visits (back pain) & 0.00 \\
\hline Number of visits (mood disorders) & 0.00 & Number of visits (skin conditions) & 0.00 \\
\hline Number ambulatory-care-sensitive visits & 0.00 & Pre-lottery SNAP recipient & 0.00 \\
\hline Number of visits (skin conditions) & 0.00 & Number ambulatory-care-sensitive visits & 0.00 \\
\hline Number of visits (abdominal pain) & 0.00 & Number of visits (headache) & 0.00 \\
\hline Number of visits (back pain) & 0.00 & Number of visits (mood disorders) & 0.00 \\
\hline Pre-lottery TANF recipient & 0.00 & Signed up self for lottery & 0.00 \\
\hline Number of visits (chest pain) & 0.00 & Pre-lottery TANF recipient & 0.00 \\
\hline Number of visits (headache) & 0.00 & English as preferred language & 0.00 \\
\hline Provided P.O. box address & 0.00 & Provided P.O. box address & 0.00 \\
\hline
\end{tabular}

Notes: This table shows the top variable importance scores of all characteristics for growing the generalized random forests used to estimate the ITE of Medicaid coverage for overall ED visits. The variable importance measure is a simple weighted sum of the proportion of times a variable is used in a splitting step at each depth in growing the causal forest, thus, capturing how important a variable is for driving treatment effect heterogeneity. The sample consists of 24,615 individuals in the Taubman et al. (2014) sample with non-missing information on pre-lottery emergency department utilization and SNAP/TANF receipt. 
Table A.2: Group average treatment effects of Medicaid on the number of ED visits

\begin{tabular}{|c|c|c|c|c|}
\hline \multirow[b]{2}{*}{ Group } & \multicolumn{3}{|c|}{ GRF estimates } & \multirow[b]{2}{*}{$\% \mathrm{~N}$} \\
\hline & GATE & SE & $p$-value & \\
\hline Female: & 0.27 & 0.16 & 0.08 & 0.55 \\
\hline Male: & 0.43 & 0.17 & 0.01 & 0.45 \\
\hline Gave phone number: No & -0.06 & 0.33 & 0.86 & 0.13 \\
\hline Gave phone number: Yes & 0.42 & 0.12 & 0.00 & 0.87 \\
\hline English as preferred language: No & 0.14 & 0.17 & 0.41 & 0.14 \\
\hline English as preferred language: Yes & 0.39 & 0.13 & 0.00 & 0.86 \\
\hline First week sign-up: No & 0.38 & 0.14 & 0.01 & 0.62 \\
\hline First week sign-up: Yes & 0.29 & 0.19 & 0.12 & 0.38 \\
\hline Pre-lottery SNAP recipient: No & 0.21 & 0.15 & 0.16 & 0.46 \\
\hline Pre-lottery SNAP recipient: Yes & 0.45 & 0.17 & 0.01 & 0.54 \\
\hline Pre-lottery TANF recipient: No & 0.34 & 0.11 & 0.00 & 0.98 \\
\hline Pre-lottery TANF recipient: Yes & 0.93 & 1.21 & 0.44 & 0.02 \\
\hline Age $\geq 50:$ No & 0.43 & 0.14 & 0.00 & 0.75 \\
\hline Age $\geq 50:$ Yes & 0.05 & 0.20 & 0.81 & 0.25 \\
\hline Two+ household members on lottery list: No & 0.32 & 0.14 & 0.02 & 0.80 \\
\hline Two+ household members on lottery list: Yes & 0.52 & 0.16 & 0.00 & 0.20 \\
\hline Any pre-lottery ED visit No & 0.22 & 0.09 & 0.01 & 0.69 \\
\hline Any pre-lottery ED visit: Yes & 0.67 & 0.32 & 0.03 & 0.31 \\
\hline Any pre-lottery on-hours ED visit: No & 0.19 & 0.09 & 0.04 & 0.77 \\
\hline Any pre-lottery on-hours ED visit: Yes & 0.87 & 0.41 & 0.03 & 0.23 \\
\hline Any pre-lottery off-hours ED visit: No & 0.22 & 0.09 & 0.02 & 0.81 \\
\hline Any pre-lottery off-hours ED visit: Yes & 0.95 & 0.45 & 0.04 & 0.19 \\
\hline Any pre-lottery emergent, non-preventable ED visit: No & 0.22 & 0.09 & 0.02 & 0.87 \\
\hline Any pre-lottery emergent, non-preventable ED visit: Yes & 1.15 & 0.63 & 0.07 & 0.13 \\
\hline Any pre-lottery emergent, preventable ED visit: No & 0.25 & 0.09 & 0.01 & 0.92 \\
\hline Any pre-lottery emergent, preventable ED visit: Yes & 1.50 & 0.96 & 0.12 & 0.08 \\
\hline Any pre-lottery primary care treatable ED visit: No & 0.18 & 0.09 & 0.04 & 0.81 \\
\hline Any pre-lottery primary care treatable ED visit: Yes & 1.03 & 0.46 & 0.03 & 0.19 \\
\hline Any pre-lottery non-emergent ED visit: No & 0.27 & 0.09 & 0.00 & 0.86 \\
\hline Any pre-lottery non-emergent ED visit: Yes & 0.82 & 0.61 & 0.18 & 0.14 \\
\hline
\end{tabular}

Notes: This table reports the group average treatment effects of Medicaid based on generalized random forests. The baseline sample consists of 24,615 individuals in the Taubman et al. (2014) sample with non-missing information on pre-lottery emergency department utilization and SNAP/TANF receipt. 


\section{Table A.3: Characteristics of individuals who increased and decreased ED use (Number of visits)}

\begin{tabular}{|c|c|c|c|}
\hline Variable & $\begin{array}{c}\text { Increased } \\
\text { ED use }\end{array}$ & $\begin{array}{l}\text { Decreased } \\
\text { ED use }\end{array}$ & Difference \\
\hline \multicolumn{4}{|l|}{ Lottery list characteristics } \\
\hline Age (years) & 38.54 & 42.88 & $-4.34 * * *$ \\
\hline Gave phone number & 0.86 & 0.89 & $-0.03 * * *$ \\
\hline English as preferred language & 0.87 & 0.84 & $0.03 * * *$ \\
\hline Female & 0.53 & 0.58 & $-0.05 * * *$ \\
\hline Week of lottery sign up & 1.59 & 1.56 & 0.03 \\
\hline Provided P.O. box address & 0.02 & 0.03 & $-0.01 * * *$ \\
\hline Signed up self for lottery & 0.89 & 0.92 & $-0.03 * * *$ \\
\hline Pre-lottery SNAP recipient & 0.59 & 0.36 & $0.23 * * *$ \\
\hline Pre-lottery SNAP benefit amount (\$) & 1497.29 & 817.99 & $679.30 * * *$ \\
\hline Pre-lottery TANF recipient & 0.02 & 0.02 & $0.00 * * *$ \\
\hline Pre-lottery TANF benefit amount $(\$)$ & 96.79 & 94.84 & 1.95 \\
\hline \multicolumn{4}{|l|}{ Pre-lottery ED usage } \\
\hline Number of overall visits & 0.90 & 0.30 & $0.60 * * *$ \\
\hline Number of inpatient visits & 0.10 & 0.06 & $0.04 * * *$ \\
\hline Number of outpatient visits & 0.80 & 0.25 & $0.55 * * *$ \\
\hline Number of on-hours visits & 0.52 & 0.19 & $0.33 * * *$ \\
\hline Number of off-hours visits & 0.38 & 0.11 & $0.27 * * *$ \\
\hline Number of emergent, non-preventable visits & 0.18 & 0.06 & $0.12 * * *$ \\
\hline Number of emergent, preventable visits & 0.07 & 0.03 & $0.04 * * *$ \\
\hline Number of primary care treatable visits & 0.32 & 0.08 & $0.24 * * *$ \\
\hline Number of non-emergent visits & 0.18 & 0.07 & $0.11 * * *$ \\
\hline Number ambulatory-care-sensitive visits & 0.05 & 0.03 & $0.02 * * *$ \\
\hline Number of visits (chronic conditions) & 0.15 & 0.07 & $0.08 * * *$ \\
\hline Number of visits (injury) & 0.20 & 0.05 & $0.15 * * *$ \\
\hline Number of visits (skin conditions) & 0.06 & 0.01 & $0.05 * * *$ \\
\hline Number of visits (abdominal pain) & 0.04 & 0.01 & $0.03 * * *$ \\
\hline Number of visits (back pain) & 0.04 & 0.02 & $0.02 * * *$ \\
\hline Number of visits (chest pain) & 0.02 & 0.01 & $0.01 * * *$ \\
\hline Number of visits (headache) & 0.03 & 0.01 & $0.02 * * *$ \\
\hline Number of visits (mood disorders) & 0.03 & 0.01 & $0.02 * * *$ \\
\hline Number of visits (psychiatric conditions) & 0.07 & 0.03 & $0.04 * * *$ \\
\hline Sum of total ED charges & 1027.60 & 387.86 & $639.74 * * *$ \\
\hline $\mathrm{N}$ & 18581.00 & 6018.00 & 24599 \\
\hline
\end{tabular}

Notes: This table reports the means of individual characteristics and pre-randomization ED use for those estimated to increase and decrease ED use upon receiving Medicaid coverage based on the causal forest CATE estimates. ED use is measured as the number of ED visits. Panel A reports the means for the full sample while Panel B is limited to effects significant at the $10 \%$ level. The sample consists of 24,615 individuals in the Taubman et al. (2014) sample with nonmissing information on pre-lottery emergency department utilization and SNAP/TANF receipt. 



\section{B. Supplementary Material for Intent-to-treat effects}

Figure B.1: Distribution of individualized treatment effects of winning the lottery on the propensity of ED use

(a) Any visit

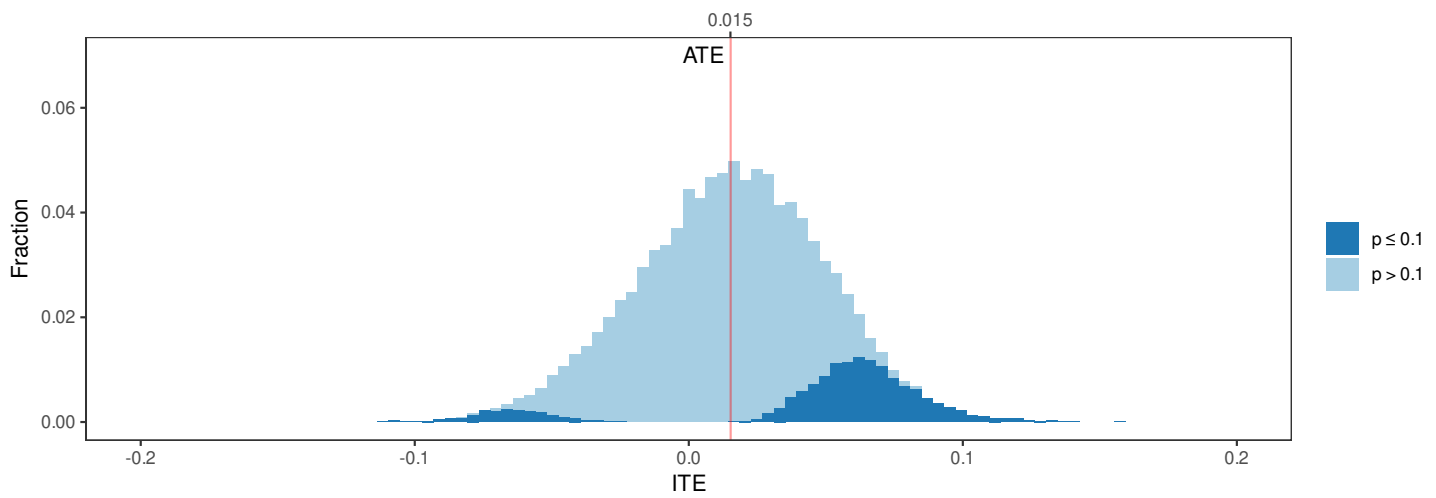

(b) Outpatient

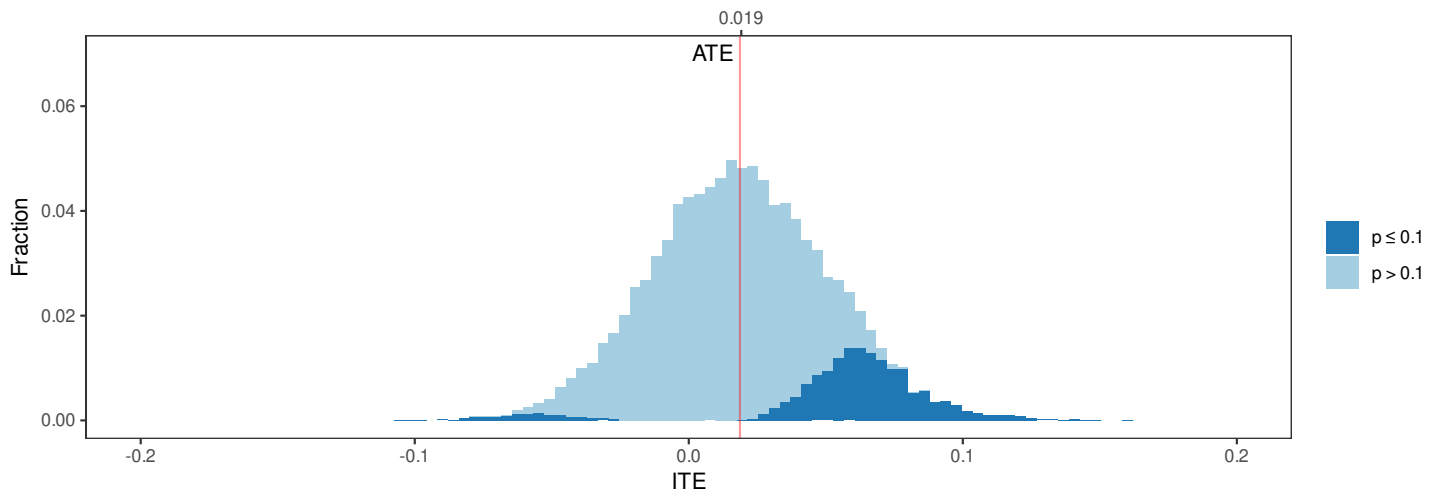

(c) Inpatient

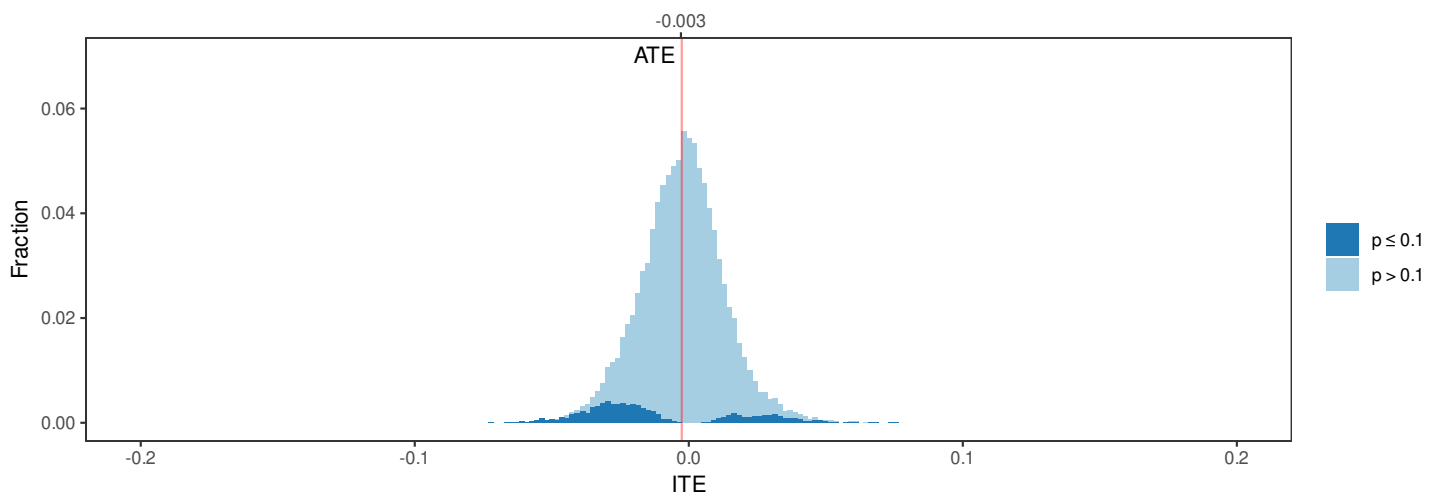

Notes: This figure plots the individualized treatment effects of winning the lottery (and being invited to apply for Medicaid) on any overall ED visit (panel a), any outpatient ED visits (panel b), and any inpatient ED use (panel c) based on generalized random forests. The darker shade denotes statistical significance at the $10 \%$ level. The red vertical line indicates the local average treatment effect. The baseline sample consists of 24,615 individuals in the Taubman et al. (2014) sample with non-missing information on pre-lottery emergency department utilization and SNAP/TANF receipt. The estimates displayed exclude less than half a percentile at the top and bottom of the distribution, resulting in the axes corresponding approximately to the percentile range $[0.5 \%, 99.5 \%]$. Bin size is chosen according to the Freedman-Diaconis rule. 


\section{Figure B.2: Distribution of individualized treatment effects of winning the lottery on the propensity of ED use by type of condition}

(a) Emergent, non-preventable

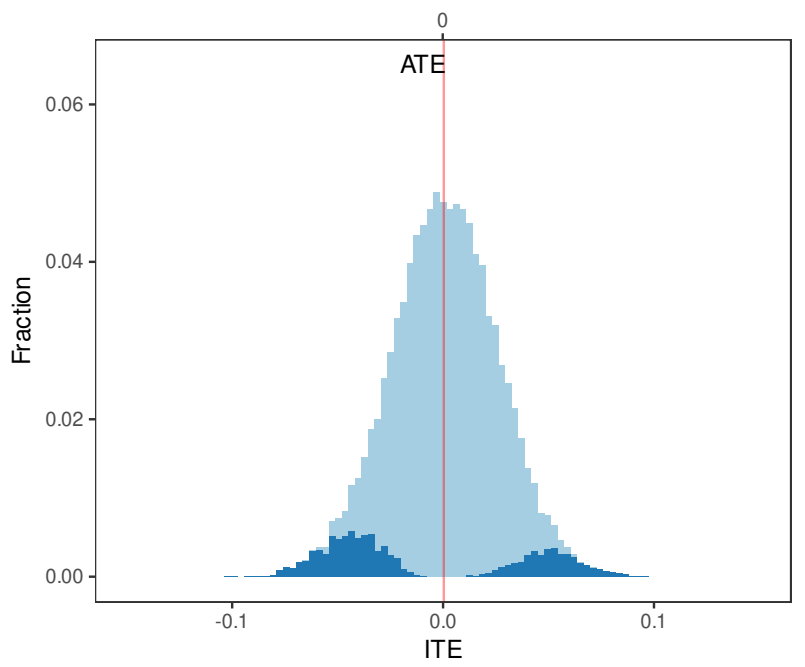

(c) Primary care treatable

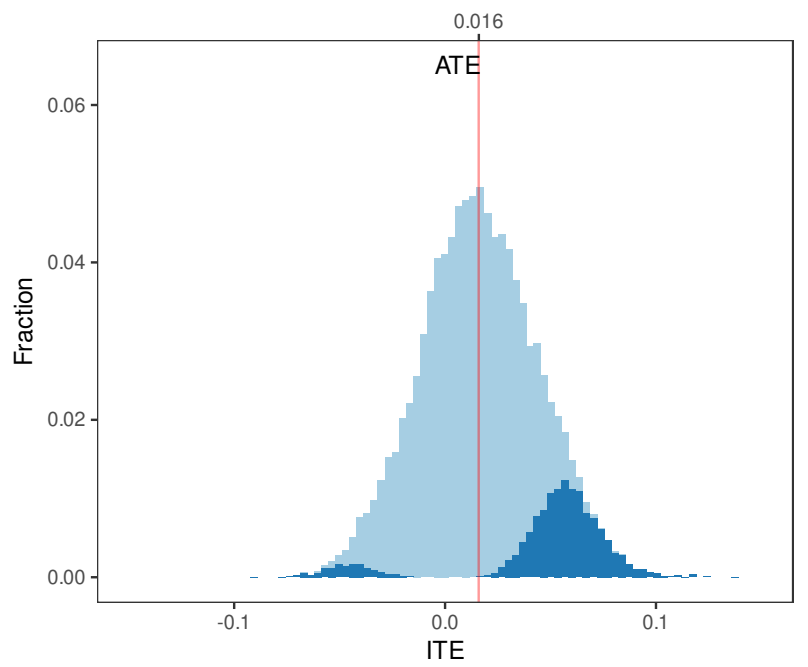

(b) Emergent, preventable

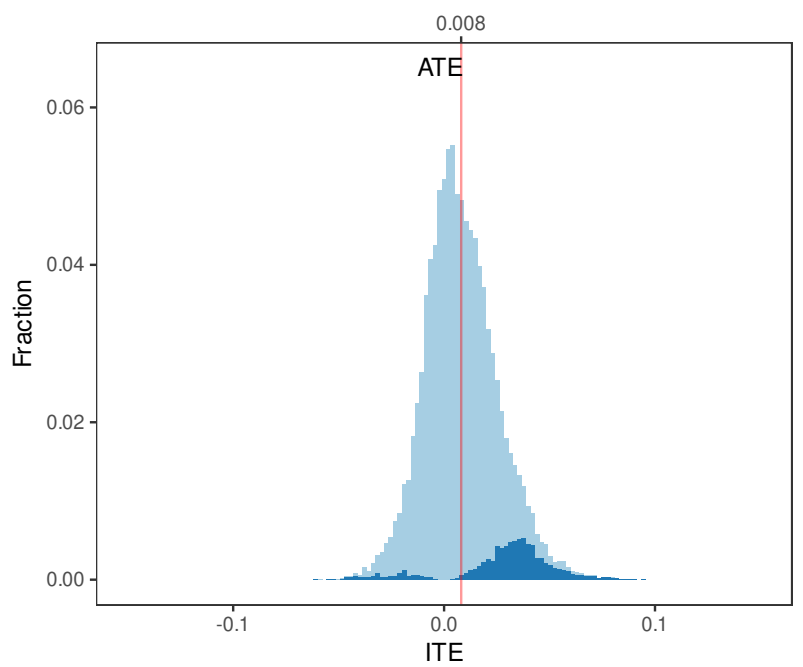

(d) Non-emergent

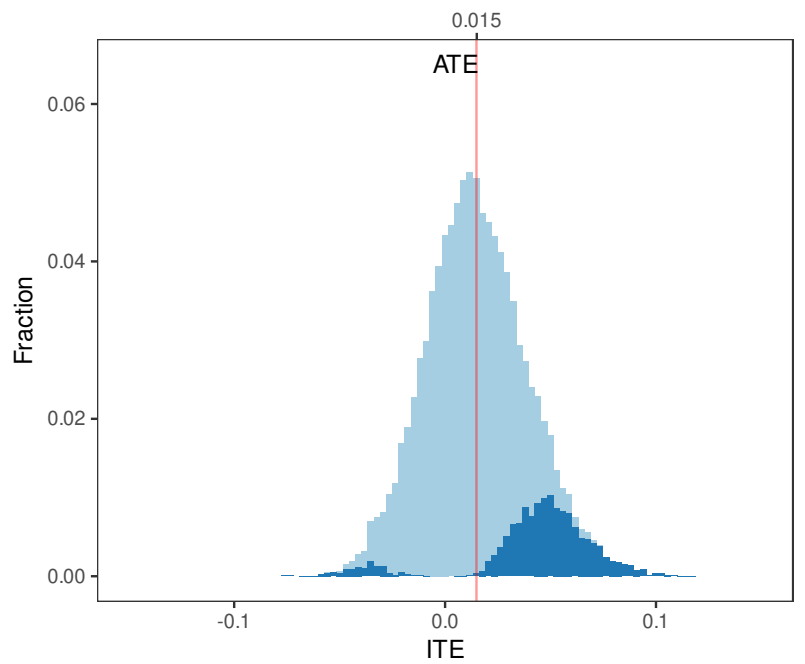

$p \leq 0.1$ $p>0.1$

Notes: This figure plots the individualized treatment effects of winning the lottery (and being invited to apply for Medicaid) by type of ED visit based on generalized random forests for any emergent, non-preventable visit (panel a), any emergent, preventable visit (panel b), any primary care treatable visit (panel c), and any non-emergent visit (panel d). Measures of the type of ED visit are based on Billings et al.'s (2000) algorithm described in Taubman et al. (2014). We use these measures to construct binary indicators of ED visits by type of condition as described in the main text. The darker shade denotes statistical significance at the $10 \%$ level. The red vertical line indicates the local average treatment effect. The baseline sample consists of 24,615 individuals in the Taubman et al. (2014) sample with non-missing information on pre-lottery emergency department utilization and SNAP/TANF receipt. The estimates displayed exclude less than half a percentile at the top and bottom of the distribution, resulting in the axes corresponding approximately to the percentile range $[0.5 \%, 99.5 \%]$. Bin size is chosen according to the Freedman-Diaconis rule. 
Figure B.3: Variable importance scores in growing causal forest (Any visit)

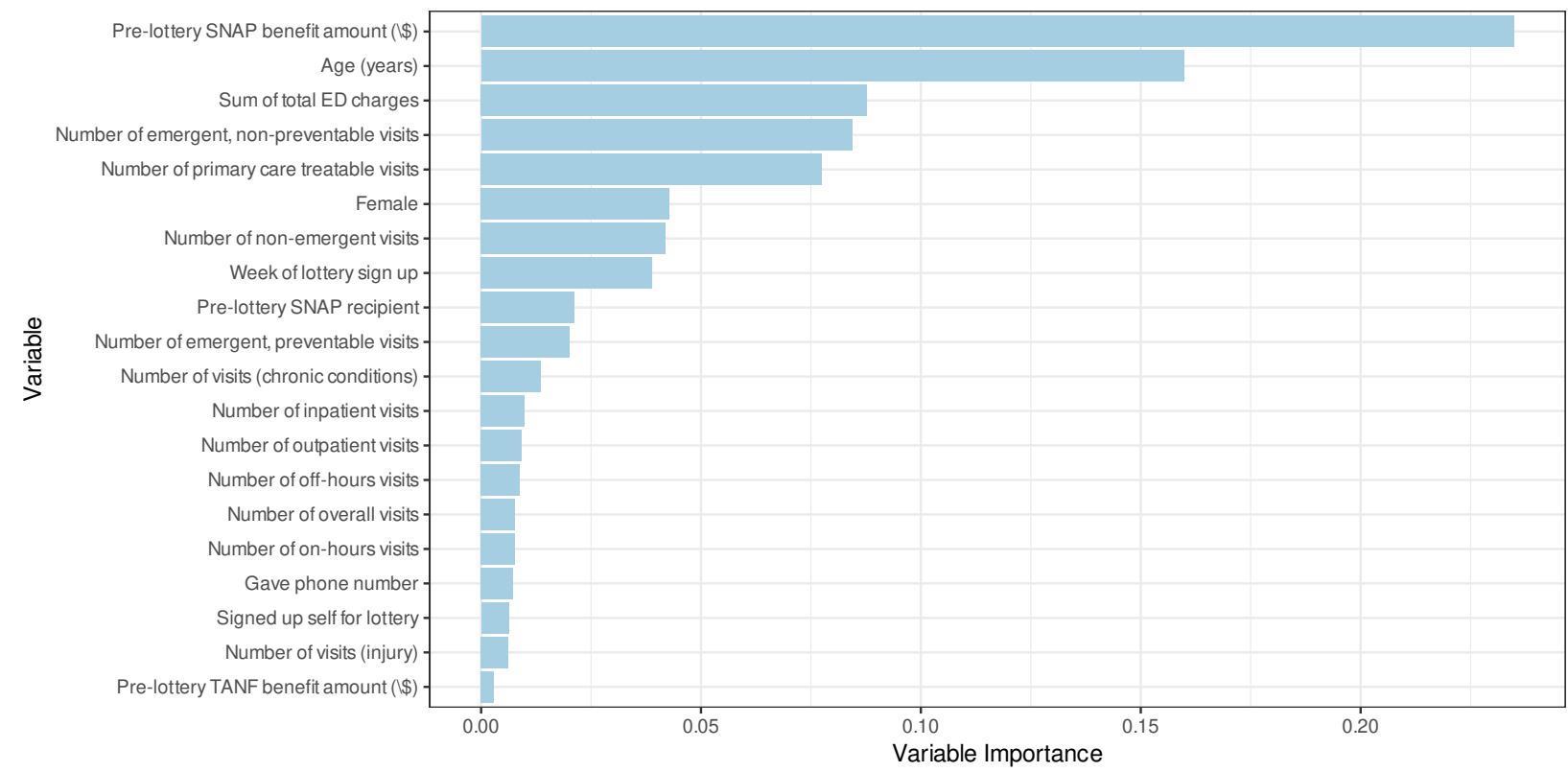

Notes This figure shows the estimates of variable importance for the top 20 characteristics used in growing the generalized random forests in estimating the CATE of winning the lottery (and being invited to apply for Medicaid) for any overall ED visit. The variable importance measure is a simple weighted sum of the proportion of times a variable is used in a splitting step at each depth in growing the causal forest, thus, capturing how important a variable is for driving treatment effect heterogeneity. The sample consists of 24,615 individuals in the Taubman et al. (2014) sample with non-missing information on pre-lottery emergency department utilization and SNAP/TANF receipt. 


\section{Figure B.4: Distribution of individualized treatment effects of winning the lottery on the number of ED visits}

(a) Any visit

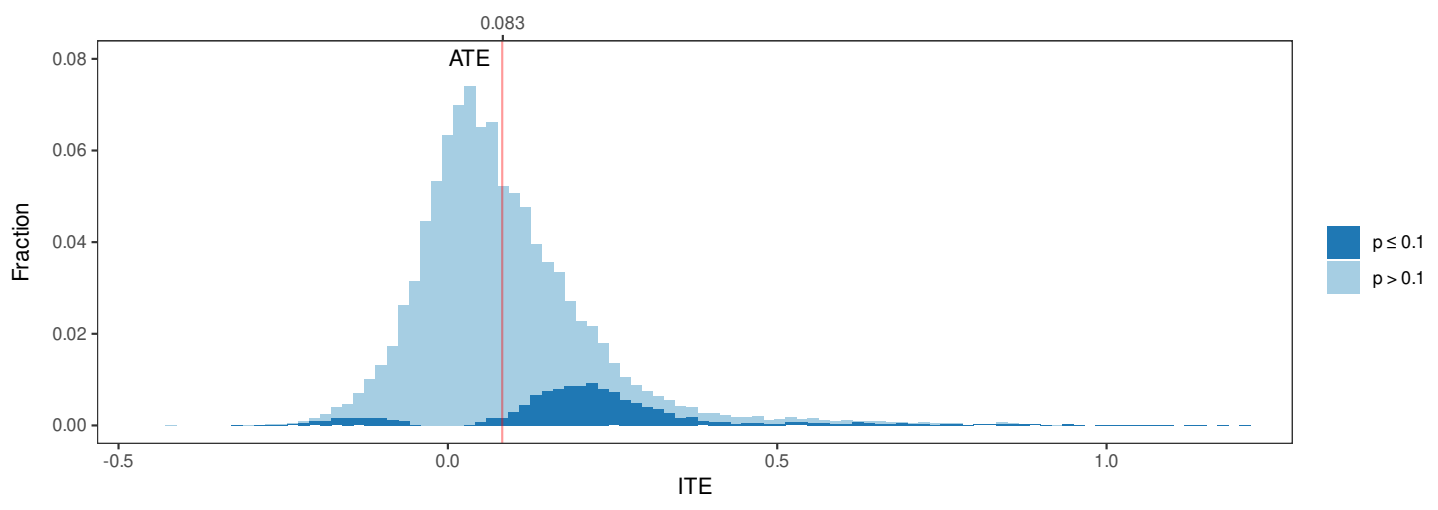

(b) Outpatient

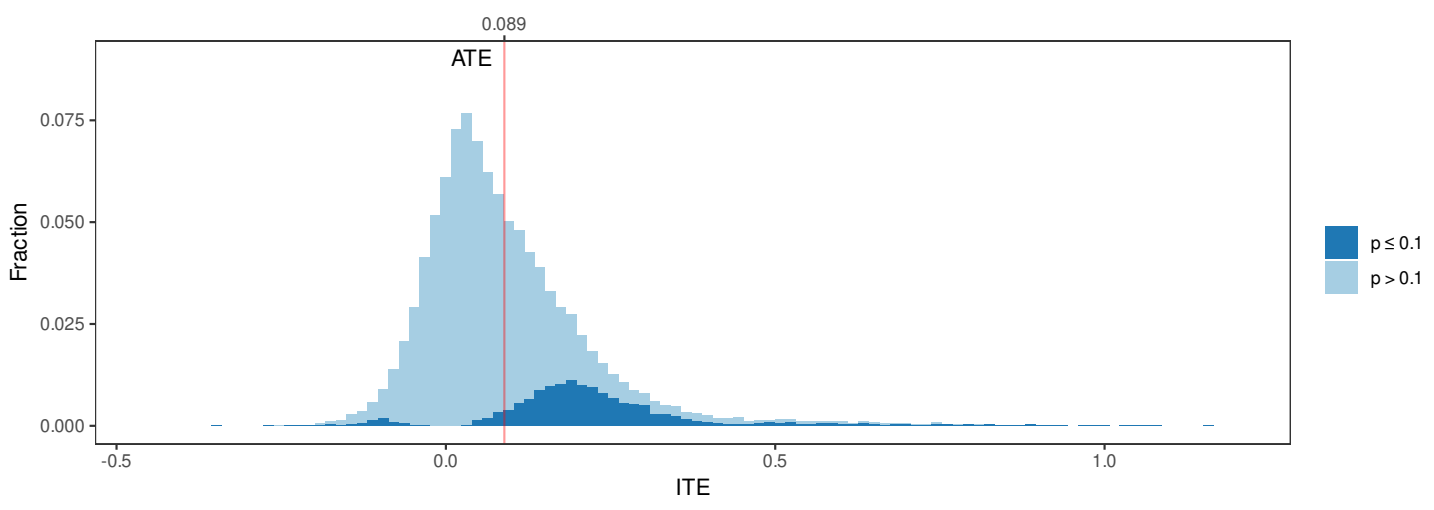

(c) Inpatient

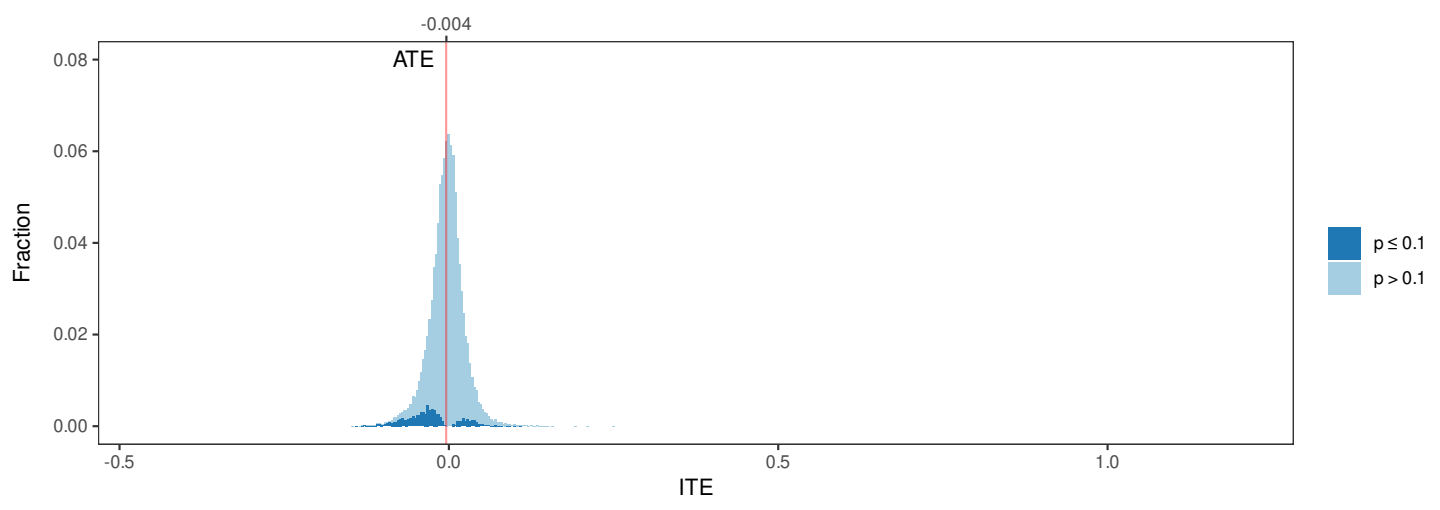

Notes: This figure plots the individualized treatment effects of winning the lottery (and being invited to apply for Medicaid) on the number of overall ED visit (panel a), the number of outpatient ED visits (panel b), and the number of inpatient ED use (panel c) based on generalized random forests. The darker shade denotes statistical significance at the $10 \%$ level. The red vertical line indicates the local average treatment effect. The baseline sample consists of 24,615 individuals in the Taubman et al. (2014) sample with non-missing information on pre-lottery emergency department utilization and SNAP/TANF receipt. The estimates displayed exclude less than half a percentile at the top and bottom of the distribution, resulting in the axes corresponding approximately to the percentile range [0.5\%, 99.5\%]. Bin size is chosen according to the Freedman-Diaconis rule. 


\section{Figure B.5: Distribution of individualized treatment effects of winning the lottery on the number of ED visits by type of condition}

(a) Emergent, non-preventable

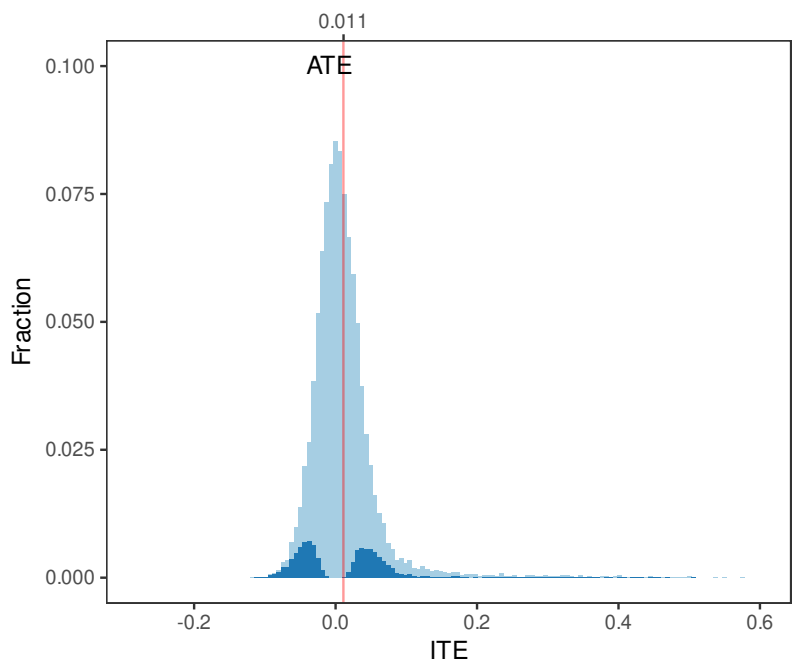

(c) Primary care treatable

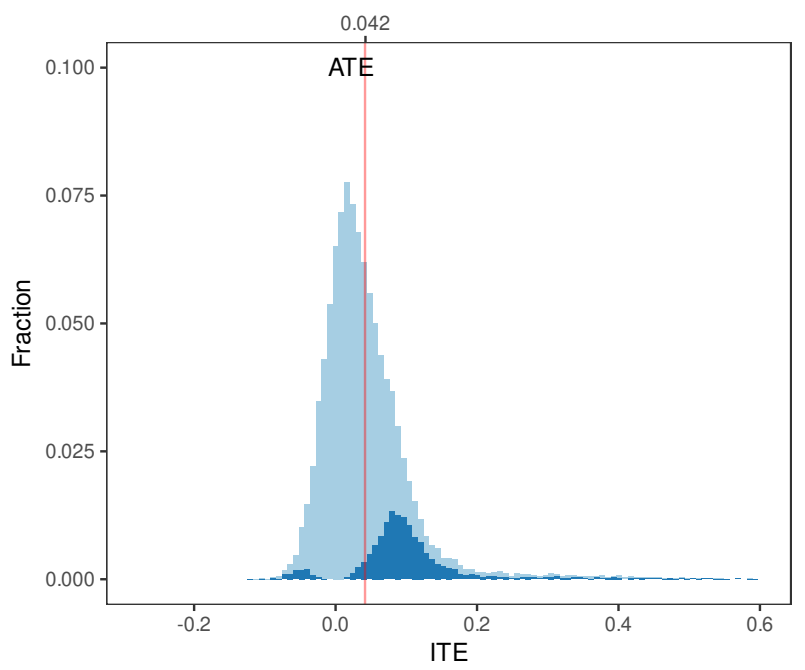

(b) Emergent, preventable

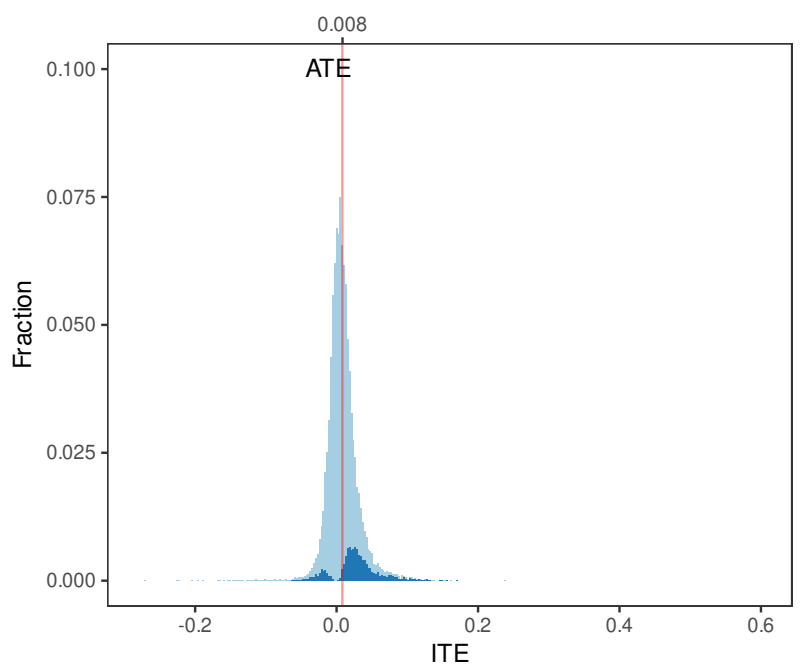

(d) Non-emergent

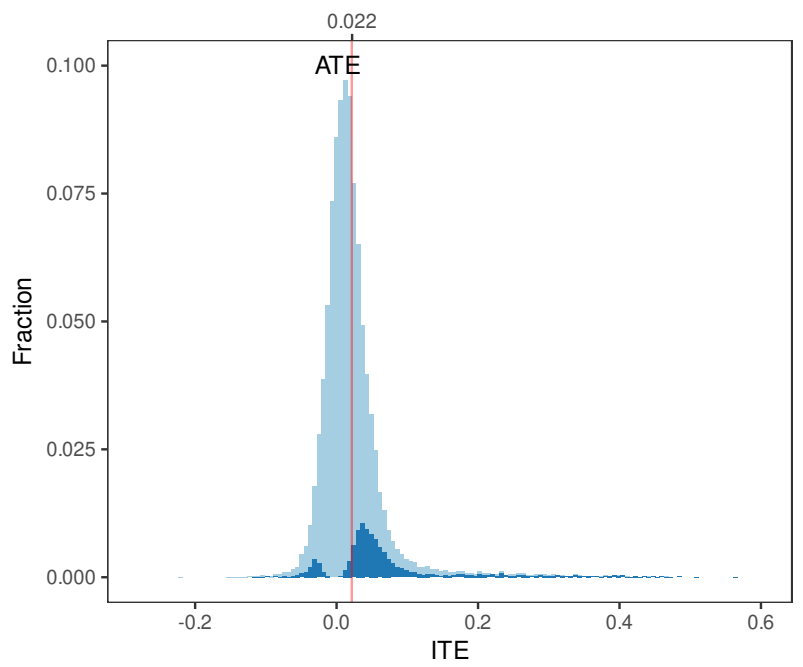

$p \leq 0.1$ $p>0.1$

Notes: This figure plots the individualized treatment effects of winning the lottery (and being invited to apply for Medicaid) by type of ED visit based on generalized random forests for the number of emergent, non-preventable visit (panel a), the number of emergent, preventable visit (panel b), the number of primary care treatable visit (panel c), and the number of non-emergent visit (panel d). Measures of the type of ED visit are based on Billings et al.'s (2000) algorithm described in Taubman et al. (2014). The darker shade denotes statistical significance at the $10 \%$ level. The red vertical line indicates the local average treatment effect. The baseline sample consists of 24,615 individuals in the Taubman et al. (2014) sample with non-missing information on pre-lottery emergency department utilization and SNAP/TANF receipt. The estimates displayed exclude less than half a percentile at the top and bottom of the distribution, resulting in the axes corresponding approximately to the percentile range $[0.5 \%, 99.5 \%]$. Bin size is chosen according to the Freedman-Diaconis rule. 


\section{Figure B.6: Variable importance scores in growing causal forest (Number of visits)}

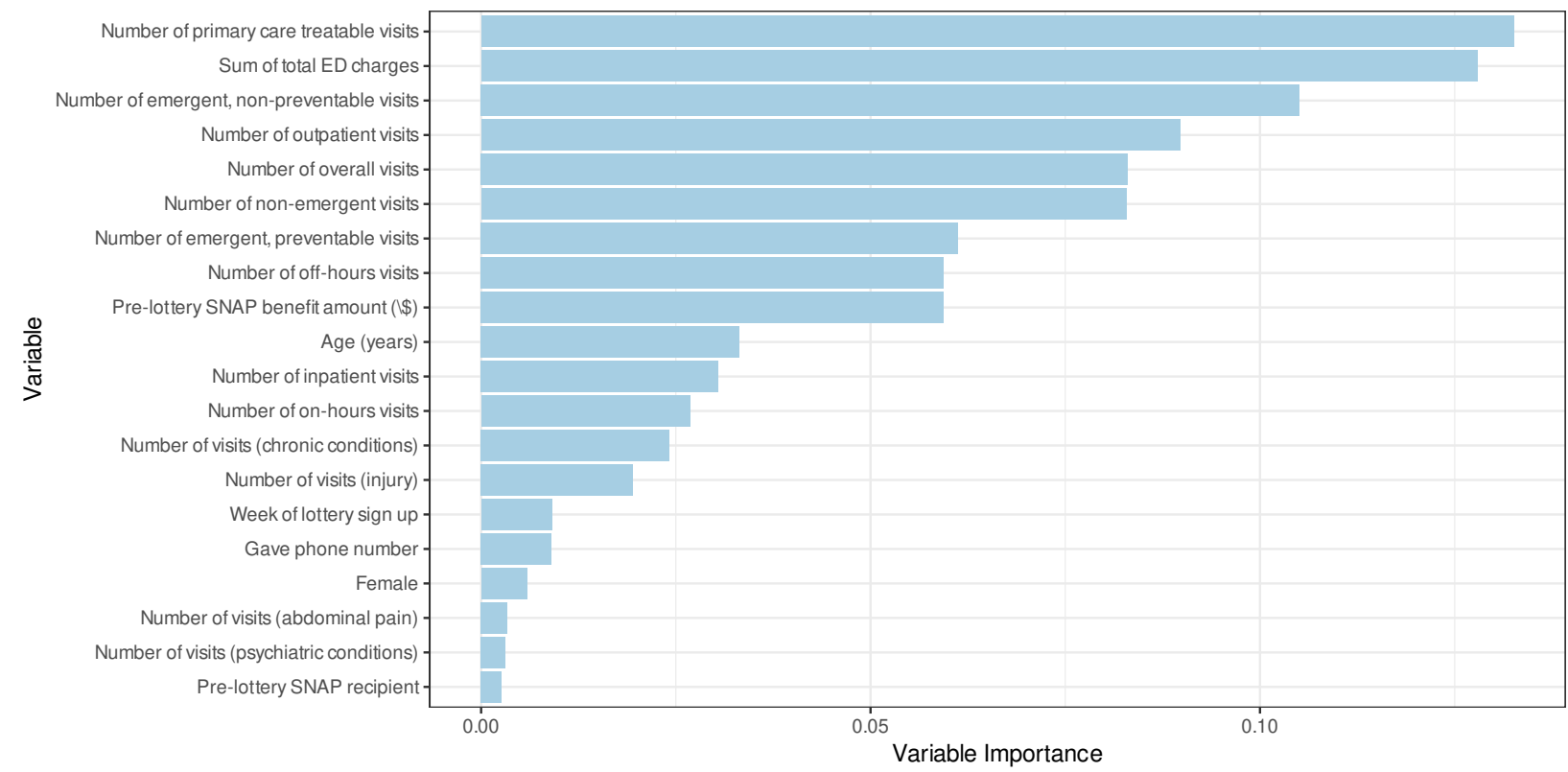

Notes This figure shows the estimates of variable importance for the top 20 characteristics used in growing the generalized random forests in estimating the CATE of winning the lottery (and being invited to apply for Medicaid) for the number of overall ED visits. The variable importance measure is a simple weighted sum of the proportion of times a variable is used in a splitting step at each depth in growing the causal forest, thus, capturing how important a variable is for driving treatment effect heterogeneity. The sample consists of 24,615 individuals in the Taubman et al. (2014) sample with non-missing information on pre-lottery emergency department utilization and SNAP/TANF receipt. 
Table B.1: Treatment effect estimates of winning the lottery on ED use

\begin{tabular}{|c|c|c|c|c|c|c|}
\hline \multirow[b]{2}{*}{ Variable } & \multicolumn{3}{|c|}{ GRF estimates } & \multicolumn{3}{|c|}{ Linear estimates } \\
\hline & ATE & SE & $p$-value & ATE & SE & $p$-value \\
\hline \multicolumn{7}{|l|}{ Extensive margin } \\
\hline Any overall visit & 0.015 & 0.006 & 0.008 & 0.017 & 0.006 & 0.004 \\
\hline Any inpatient visit & -0.003 & 0.003 & 0.430 & -0.003 & 0.003 & 0.424 \\
\hline Any outpatient visit & 0.019 & 0.006 & 0.001 & 0.020 & 0.006 & 0.001 \\
\hline Any emergent, non-preventable visit & 0.000 & 0.005 & 0.940 & 0.002 & 0.005 & 0.731 \\
\hline Any emergent, preventable visit & 0.008 & 0.004 & 0.026 & 0.010 & 0.004 & 0.010 \\
\hline Any primary care treatable visit & 0.016 & 0.005 & 0.002 & 0.017 & 0.005 & 0.001 \\
\hline Any non-emergent visit & 0.015 & 0.005 & 0.001 & 0.016 & 0.005 & 0.001 \\
\hline \multicolumn{7}{|l|}{ Intensive margin } \\
\hline Number of overall visits & 0.083 & 0.027 & 0.002 & 0.093 & 0.026 & 0.000 \\
\hline Number of inpatient visits & -0.004 & 0.006 & 0.519 & -0.004 & 0.006 & 0.516 \\
\hline Number of outpatient visits & 0.089 & 0.024 & 0.000 & 0.096 & 0.024 & 0.000 \\
\hline Number of emergent, non-preventable visits & 0.011 & 0.008 & 0.162 & 0.010 & 0.008 & 0.224 \\
\hline Number of emergent, preventable visits & 0.008 & 0.004 & 0.056 & 0.009 & 0.004 & 0.034 \\
\hline Number of primary care treatable visits & 0.042 & 0.011 & 0.000 & 0.042 & 0.011 & 0.000 \\
\hline Number of non-emergent visits & 0.022 & 0.008 & 0.007 & 0.026 & 0.008 & 0.002 \\
\hline
\end{tabular}

Notes: This table reports the estimates of winning the lottery on ED use based on generalized random forests and a linear model. The sample consists of 24,615 individuals in the Taubman et al. (2014) sample with nonmissing information on pre-lottery emergency department utilization and SP/TANF receipt.

\section{Table B.2: Empirical quantiles of the distribution of individualized treatment effects of winning the lottery on ED use}

\begin{tabular}{|c|c|c|c|c|c|c|}
\hline Variable & ATE & Min & $25 \%$ & $50 \%$ & $75 \%$ & Max \\
\hline \multicolumn{7}{|l|}{ Extensive margin } \\
\hline Any overall visit & 0.015 & -0.112 & -0.007 & 0.017 & 0.039 & 0.159 \\
\hline Any inpatient visit & -0.003 & -0.072 & -0.012 & -0.002 & 0.007 & 0.076 \\
\hline Any outpatient visit & 0.019 & -0.107 & -0.003 & 0.018 & 0.041 & 0.159 \\
\hline Any emergent, non-preventable visit & 0.000 & -0.103 & -0.016 & 0.001 & 0.018 & 0.096 \\
\hline Any emergent, preventable visit & 0.008 & -0.061 & -0.003 & 0.007 & 0.019 & 0.095 \\
\hline Any primary care treatable visit & 0.016 & -0.089 & -0.003 & 0.016 & 0.035 & 0.137 \\
\hline Any non-emergent visit & 0.015 & -0.077 & -0.001 & 0.014 & 0.031 & 0.116 \\
\hline \multicolumn{7}{|l|}{ Intensive margin } \\
\hline Number of overall visits & 0.083 & -0.427 & 0.000 & 0.061 & 0.145 & 1.205 \\
\hline Number of inpatient visits & -0.004 & -0.148 & -0.018 & -0.003 & 0.011 & 0.248 \\
\hline Number of outpatient visits & 0.089 & -0.347 & 0.009 & 0.065 & 0.146 & 1.154 \\
\hline Number of emergent, non-preventable visits & 0.011 & -0.116 & -0.015 & 0.004 & 0.026 & 0.576 \\
\hline Number of emergent, preventable visits & 0.008 & -0.270 & -0.003 & 0.006 & 0.018 & 0.238 \\
\hline Number of primary care treatable visits & 0.042 & -0.118 & 0.003 & 0.031 & 0.067 & 0.686 \\
\hline Number of non-emergent visits & 0.022 & -0.218 & -0.002 & 0.014 & 0.034 & 0.563 \\
\hline
\end{tabular}

Notes: This table reports selected quantiles of the individualized treatment effects of winning the lottery (and being invited to apply for Medicaid) on ED use based on generalized random forests. The first column reports the average effect (intent-to-treat effect). The baseline sample consists of 24,615 individuals in the Taubman et al. (2014) sample with non-missing information on pre-lottery emergency department utilization and SNAP/TANF receipt. 
Table B.3: GATE estimates of winning the lottery on the propensity of ED use

\begin{tabular}{|c|c|c|c|c|c|c|c|}
\hline \multirow[b]{2}{*}{ Group } & \multicolumn{3}{|c|}{ Panel A: GRF estimates } & \multicolumn{3}{|c|}{ Panel B: Linear estimates } & \multirow[b]{2}{*}{$\% \mathrm{~N}$} \\
\hline & GATE & SE & $p$-value & GATE & SE & $p$-value & \\
\hline ATE & 0.02 & 0.01 & 0.01 & 0.02 & 0.01 & 0.00 & 100.00 \\
\hline Female: & 0.00 & 0.01 & 0.72 & 0.01 & 0.01 & 0.34 & 0.55 \\
\hline Male: & 0.03 & 0.01 & 0.00 & 0.03 & 0.01 & 0.00 & 0.45 \\
\hline Gave phone number: No & 0.01 & 0.02 & 0.54 & 0.01 & 0.02 & 0.66 & 0.13 \\
\hline Gave phone number: Yes & 0.02 & 0.01 & 0.01 & 0.02 & 0.01 & 0.00 & 0.87 \\
\hline English as preferred language: No & 0.01 & 0.01 & 0.59 & 0.00 & 0.01 & 0.73 & 0.14 \\
\hline English as preferred language: Yes & 0.02 & 0.01 & 0.01 & 0.02 & 0.01 & 0.01 & 0.86 \\
\hline First week sign-up: No & 0.01 & 0.01 & 0.06 & 0.02 & 0.01 & 0.01 & 0.62 \\
\hline First week sign-up: Yes & 0.02 & 0.01 & 0.06 & 0.01 & 0.01 & 0.16 & 0.38 \\
\hline Pre-lottery SNAP recipient: No & 0.00 & 0.01 & 0.94 & 0.00 & 0.01 & 0.62 & 0.46 \\
\hline Pre-lottery SNAP recipient: Yes & 0.03 & 0.01 & 0.00 & 0.02 & 0.01 & 0.01 & 0.54 \\
\hline Pre-lottery TANF recipient: No & 0.02 & 0.01 & 0.01 & 0.02 & 0.01 & 0.00 & 0.98 \\
\hline Pre-lottery TANF recipient: Yes & -0.01 & 0.04 & 0.78 & 0.01 & 0.04 & 0.82 & 0.02 \\
\hline Age $\geq 50:$ No & 0.02 & 0.01 & 0.00 & 0.02 & 0.01 & 0.00 & 0.75 \\
\hline Age $\geq 50:$ Yes & -0.01 & 0.01 & 0.67 & 0.00 & 0.01 & 0.77 & 0.25 \\
\hline Two+ household members on lottery list: No & 0.01 & 0.01 & 0.06 & 0.01 & 0.01 & 0.06 & 0.80 \\
\hline Two+ household members on lottery list: Yes & 0.03 & 0.01 & 0.02 & 0.04 & 0.01 & 0.00 & 0.20 \\
\hline Any pre-lottery ED visit No & 0.01 & 0.01 & 0.07 & 0.02 & 0.01 & 0.02 & 0.69 \\
\hline Any pre-lottery ED visit: Yes & 0.02 & 0.01 & 0.05 & 0.02 & 0.01 & 0.09 & 0.31 \\
\hline Any pre-lottery on-hours ED visit: No & 0.01 & 0.01 & 0.03 & 0.02 & 0.01 & 0.01 & 0.77 \\
\hline Any pre-lottery on-hours ED visit: Yes & 0.02 & 0.01 & 0.11 & 0.02 & 0.01 & 0.13 & 0.23 \\
\hline Any pre-lottery off-hours ED visit: No & 0.01 & 0.01 & 0.04 & 0.01 & 0.01 & 0.03 & 0.81 \\
\hline Any pre-lottery off-hours ED visit: Yes & 0.02 & 0.01 & 0.08 & 0.02 & 0.01 & 0.16 & 0.19 \\
\hline Any pre-lottery emergent, non-preventable ED visit: No & 0.01 & 0.01 & 0.07 & 0.01 & 0.01 & 0.03 & 0.79 \\
\hline Any pre-lottery emergent, non-preventable ED visit: Yes & 0.03 & 0.01 & 0.02 & 0.03 & 0.01 & 0.07 & 0.21 \\
\hline Any pre-lottery emergent, preventable ED visit: No & 0.01 & 0.01 & 0.02 & 0.02 & 0.01 & 0.00 & 0.90 \\
\hline Any pre-lottery emergent, preventable ED visit: Yes & 0.03 & 0.02 & 0.15 & 0.03 & 0.02 & 0.13 & 0.10 \\
\hline Any pre-lottery primary care treatable ED visit: No & 0.01 & 0.01 & 0.10 & 0.01 & 0.01 & 0.03 & 0.74 \\
\hline Any pre-lottery primary care treatable ED visit: Yes & 0.03 & 0.01 & 0.02 & 0.03 & 0.01 & 0.05 & 0.26 \\
\hline Any pre-lottery non-emergent ED visit: No & 0.01 & 0.01 & 0.04 & 0.02 & 0.01 & 0.01 & 0.86 \\
\hline Any pre-lottery non-emergent ED visit: Yes & 0.03 & 0.02 & 0.07 & 0.02 & 0.02 & 0.18 & 0.14 \\
\hline
\end{tabular}

Notes: This table reports the GATE estimates of winning the lottery based on generalized random forests in Panel A and the linear model GATEs in Panel B. The overall effect is reproduced in the first row. The sample consists of 24,615 individuals in the Taubman et al. (2014) sample with non-missing information on pre-lottery emergency department utilization and SNAP/TANF receipt. 
Table B.4: GATE estimates of winning the lottery on the number of ED visits

\begin{tabular}{|c|c|c|c|c|c|c|c|}
\hline \multirow[b]{2}{*}{ Group } & \multicolumn{3}{|c|}{ Panel A: GRF estimates } & \multicolumn{3}{|c|}{ Panel B: Linear estimates } & \multirow[b]{2}{*}{$\% \mathrm{~N}$} \\
\hline & GATE & SE & $p$-value & GATE & SE & $p$-value & \\
\hline ATE & 0.08 & 0.03 & 0.00 & 0.08 & 0.03 & 0.01 & 100.00 \\
\hline Female: & 0.05 & 0.03 & 0.19 & 0.05 & 0.04 & 0.21 & 0.55 \\
\hline Male: & 0.13 & 0.04 & 0.00 & 0.12 & 0.05 & 0.01 & 0.45 \\
\hline Gave phone number: No & -0.04 & 0.08 & 0.62 & -0.06 & 0.09 & 0.50 & 0.13 \\
\hline Gave phone number: Yes & 0.10 & 0.03 & 0.00 & 0.10 & 0.03 & 0.00 & 0.87 \\
\hline English as preferred language: No & 0.03 & 0.03 & 0.43 & 0.01 & 0.04 & 0.78 & 0.14 \\
\hline English as preferred language: Yes & 0.09 & 0.03 & 0.00 & 0.09 & 0.04 & 0.01 & 0.86 \\
\hline First week sign-up: No & 0.09 & 0.03 & 0.00 & 0.11 & 0.04 & 0.00 & 0.62 \\
\hline First week sign-up: Yes & 0.06 & 0.05 & 0.17 & 0.03 & 0.06 & 0.54 & 0.38 \\
\hline Pre-lottery SNAP recipient: No & 0.04 & 0.03 & 0.17 & 0.03 & 0.03 & 0.34 & 0.46 \\
\hline Pre-lottery SNAP recipient: Yes & 0.12 & 0.04 & 0.01 & 0.11 & 0.05 & 0.04 & 0.54 \\
\hline Pre-lottery TANF recipient: No & 0.08 & 0.03 & 0.00 & 0.08 & 0.03 & 0.01 & 0.98 \\
\hline Pre-lottery TANF recipient: Yes & 0.07 & 0.27 & 0.78 & 0.17 & 0.29 & 0.55 & 0.02 \\
\hline Age $\geq 50:$ No & 0.10 & 0.03 & 0.00 & 0.09 & 0.04 & 0.02 & 0.75 \\
\hline Age $\geq 50:$ Yes & 0.03 & 0.05 & 0.50 & 0.07 & 0.06 & 0.23 & 0.25 \\
\hline Two+ household members on lottery list: No & 0.07 & 0.03 & 0.02 & 0.07 & 0.04 & 0.07 & 0.80 \\
\hline Two+ household members on lottery list: Yes & 0.12 & 0.03 & 0.00 & 0.15 & 0.05 & 0.00 & 0.20 \\
\hline Any pre-lottery ED visit No & 0.05 & 0.02 & 0.01 & 0.06 & 0.02 & 0.00 & 0.69 \\
\hline Any pre-lottery ED visit: Yes & 0.15 & 0.07 & 0.04 & 0.12 & 0.09 & 0.15 & 0.31 \\
\hline Any pre-lottery on-hours ED visit: No & 0.05 & 0.02 & 0.01 & 0.06 & 0.02 & 0.00 & 0.77 \\
\hline Any pre-lottery on-hours ED visit: Yes & 0.19 & 0.09 & 0.04 & 0.17 & 0.11 & 0.12 & 0.23 \\
\hline Any pre-lottery off-hours ED visit: No & 0.05 & 0.02 & 0.02 & 0.05 & 0.02 & 0.03 & 0.81 \\
\hline Any pre-lottery off-hours ED visit: Yes & 0.22 & 0.11 & 0.04 & 0.16 & 0.12 & 0.21 & 0.19 \\
\hline Any pre-lottery emergent, non-preventable ED visit: No & 0.06 & 0.02 & 0.01 & 0.07 & 0.02 & 0.00 & 0.87 \\
\hline Any pre-lottery emergent, non-preventable ED visit: Yes & 0.23 & 0.14 & 0.11 & 0.18 & 0.17 & 0.28 & 0.13 \\
\hline Any pre-lottery emergent, preventable ED visit: No & 0.06 & 0.02 & 0.00 & 0.08 & 0.02 & 0.00 & 0.92 \\
\hline Any pre-lottery emergent, preventable ED visit: Yes & 0.30 & 0.22 & 0.17 & 0.36 & 0.25 & 0.15 & 0.08 \\
\hline Any pre-lottery primary care treatable ED visit: No & 0.05 & 0.02 & 0.03 & 0.06 & 0.02 & 0.00 & 0.81 \\
\hline Any pre-lottery primary care treatable ED visit: Yes & 0.24 & 0.11 & 0.03 & 0.19 & 0.13 & 0.13 & 0.19 \\
\hline Any pre-lottery non-emergent ED visit: No & 0.07 & 0.02 & 0.00 & 0.08 & 0.02 & 0.00 & 0.86 \\
\hline Any pre-lottery non-emergent ED visit: Yes & 0.14 & 0.14 & 0.30 & 0.07 & 0.16 & 0.68 & 0.14 \\
\hline
\end{tabular}

Notes: This table reports the GATE estimates of winning the lottery based on generalized random forests in Panel A and the GATE based on a linear model in Panel B. The overall effect is reproduced in the first row. The sample consists of 24,615 individuals in the Taubman et al. (2014) sample with non-missing information on pre-lottery emergency department utilization and SNAP/TANF receipt. 
Table B.5: Characteristics of individuals who increased and decreased ED use upon winning the lottery

\begin{tabular}{|c|c|c|c|}
\hline Variable & $\begin{array}{l}\text { Increased } \\
\text { ED use }\end{array}$ & $\begin{array}{l}\text { Decreased } \\
\text { ED use }\end{array}$ & Difference \\
\hline \multicolumn{4}{|l|}{ Lottery list characteristics } \\
\hline Age (years) & 38.81 & 41.32 & $-2.51 * * *$ \\
\hline Gave phone number & 0.87 & 0.87 & 0.00 \\
\hline English as preferred language & 0.87 & 0.84 & $0.03 * * *$ \\
\hline Female & 0.51 & 0.63 & $-0.12 * * *$ \\
\hline Week of lottery sign up & 1.57 & 1.61 & $-0.04 * *$ \\
\hline Provided P.O. box address & 0.02 & 0.03 & $-0.01 * * *$ \\
\hline Signed up self for lottery & 0.89 & 0.92 & $-0.03^{* * *}$ \\
\hline Pre-lottery SNAP recipient & 0.62 & 0.35 & $0.27 * * *$ \\
\hline Pre-lottery SNAP benefit amount (\$) & 1613.82 & 715.39 & $898.43 * * *$ \\
\hline Pre-lottery TANF recipient & 0.02 & 0.01 & $0.01 * * *$ \\
\hline Pre-lottery TANF benefit amount (\$) & 110.21 & 65.85 & $44.36^{* * *}$ \\
\hline \multicolumn{4}{|l|}{ Pre-lottery ED usage } \\
\hline Number of overall visits & 0.87 & 0.52 & $0.35^{* * *}$ \\
\hline Number of inpatient visits & 0.09 & 0.07 & $0.02 * * *$ \\
\hline Number of outpatient visits & 0.78 & 0.45 & $0.33^{* * *}$ \\
\hline Number of on-hours visits & 0.50 & 0.32 & $0.18^{* * *}$ \\
\hline Number of off-hours visits & 0.37 & 0.21 & $0.16^{* * *}$ \\
\hline Number of emergent, non-preventable visits & 0.18 & 0.10 & $0.08^{* * *}$ \\
\hline Number of emergent, preventable visits & 0.07 & 0.05 & $0.02 * * *$ \\
\hline Number of primary care treatable visits & 0.30 & 0.18 & $0.12 * * *$ \\
\hline Number of non-emergent visits & 0.18 & 0.11 & $0.07 * * *$ \\
\hline Number ambulatory-care-sensitive visits & 0.05 & 0.04 & $0.01 * * *$ \\
\hline Number of visits (chronic conditions) & 0.14 & 0.12 & $0.02 * * *$ \\
\hline Number of visits (injury) & 0.20 & 0.10 & $0.10^{* * *}$ \\
\hline Number of visits (skin conditions) & 0.05 & 0.03 & $0.02 * * *$ \\
\hline Number of visits (abdominal pain) & 0.04 & 0.02 & $0.02 * * *$ \\
\hline Number of visits (back pain) & 0.04 & 0.02 & $0.02 * * *$ \\
\hline Number of visits (chest pain) & 0.02 & 0.02 & $0.00 * * *$ \\
\hline Number of visits (headache) & 0.03 & 0.02 & $0.01 * * *$ \\
\hline Number of visits (mood disorders) & 0.02 & 0.03 & -0.01 \\
\hline Number of visits (psychiatric conditions) & 0.06 & 0.05 & 0.01 \\
\hline Sum of total ED charges & 1002.57 & 615.50 & $387.07 * * *$ \\
\hline $\mathrm{N}$ & 16871 & 7742 & 24613 \\
\hline
\end{tabular}

Notes: This table reports the means of individual characteristics and pre-randomization ED use for those estimated to increase and decrease ED use upon winning the lottery based on the causal forest CATE estimates. ED use is measured as the propensity to use the ED. Panel A reports the means for the full sample while Panel B is limited to individuals with effects significant at the $10 \%$ level. The sample consists of 24,615 individuals in the Taubman et al. (2014) sample with nonmissing information on pre-lottery emergency department utilization and SNAP/TANF receipt. 
Table B.6: Characteristics of individuals who increased and decreased ED use upon winning the lottery (Number of visits)

\begin{tabular}{|c|c|c|c|}
\hline Variable & $\begin{array}{l}\text { Increased } \\
\text { ED use }\end{array}$ & $\begin{array}{l}\text { Decreased } \\
\text { ED use }\end{array}$ & Difference \\
\hline \multicolumn{4}{|l|}{ Lottery list characteristics } \\
\hline Age (years) & 38.52 & 42.89 & $-4.37 * * *$ \\
\hline Gave phone number & 0.87 & 0.88 & $-0.01 * * *$ \\
\hline English as preferred language & 0.87 & 0.84 & $0.03 * * *$ \\
\hline Female & 0.53 & 0.59 & $-0.06 * * *$ \\
\hline Week of lottery sign up & 1.59 & 1.55 & 0.04 \\
\hline Provided P.O. box address & 0.02 & 0.03 & $-0.01 * * *$ \\
\hline Signed up self for lottery & 0.89 & 0.92 & $-0.03 * * *$ \\
\hline Pre-lottery SNAP recipient & 0.59 & 0.36 & $0.23 * * *$ \\
\hline Pre-lottery SNAP benefit amount (\$) & 1502.03 & 813.33 & $688.70 * * *$ \\
\hline Pre-lottery TANF recipient & 0.02 & 0.02 & $0.00 * *$ \\
\hline Pre-lottery TANF benefit amount (\$) & 95.68 & 98.23 & -2.55 \\
\hline \multicolumn{4}{|l|}{ Pre-lottery ED usage } \\
\hline Number of overall visits & 0.90 & 0.32 & $0.58 * * *$ \\
\hline Number of inpatient visits & 0.10 & 0.06 & $0.04 * * *$ \\
\hline Number of outpatient visits & 0.80 & 0.26 & $0.54 * * *$ \\
\hline Number of on-hours visits & 0.52 & 0.21 & $0.31 * * *$ \\
\hline Number of off-hours visits & 0.38 & 0.12 & $0.26 * * *$ \\
\hline Number of emergent, non-preventable visits & 0.18 & 0.07 & $0.11 * * *$ \\
\hline Number of emergent, preventable visits & 0.07 & 0.03 & $0.04 * * *$ \\
\hline Number of primary care treatable visits & 0.32 & 0.08 & $0.24 * * *$ \\
\hline Number of non-emergent visits & 0.18 & 0.08 & $0.10 * * *$ \\
\hline Number ambulatory-care-sensitive visits & 0.05 & 0.03 & $0.02 * * *$ \\
\hline Number of visits (chronic conditions) & 0.15 & 0.08 & $0.07 * * *$ \\
\hline Number of visits (injury) & 0.20 & 0.06 & $0.14 * * *$ \\
\hline Number of visits (skin conditions) & 0.06 & 0.01 & $0.05 * * *$ \\
\hline Number of visits (abdominal pain) & 0.04 & 0.01 & $0.03 * * *$ \\
\hline Number of visits (back pain) & 0.04 & 0.02 & $0.02 * * *$ \\
\hline Number of visits (chest pain) & 0.02 & 0.01 & $0.01 * * *$ \\
\hline Number of visits (headache) & 0.03 & 0.01 & $0.02 * * *$ \\
\hline Number of visits (mood disorders) & 0.03 & 0.02 & $0.01 * * *$ \\
\hline Number of visits (psychiatric conditions) & 0.07 & 0.03 & $0.04 * * *$ \\
\hline Sum of total ED charges & 1022.87 & 411.30 & $611.57 * * *$ \\
\hline $\mathrm{N}$ & 18494.00 & 6105.00 & 24599 \\
\hline
\end{tabular}

Notes: This table reports the means of individual characteristics and pre-randomization ED use for those estimated to increase and decrease ED use upon winning the lottery based on the causal forest CATE estimates. ED use is measured as the number of total visits. Panel A reports the means for the full sample while Panel B is limited to individuals with effects significant at the $10 \%$ level. The sample consists of 24,615 individuals in the Taubman et al. (2014) sample with non-missing information on pre-lottery emergency department utilization and SNAP/TANF receipt. 
Table B.7: Variable importance for all variables in growing causal forest (overall ED use)

\begin{tabular}{|c|c|c|c|}
\hline \multicolumn{2}{|l|}{ Any visit } & \multicolumn{2}{|l|}{ Number of visits } \\
\hline Variable & Importance & Variable & Importance \\
\hline Pre-lottery SNAP benefit amount (\$) & 0.23 & Number of primary care treatable visits & 0.13 \\
\hline Age (years) & 0.16 & Sum of total ED charges & 0.13 \\
\hline Sum of total ED charges & 0.09 & Number of emergent, non-preventable visits & 0.11 \\
\hline Number of emergent, non-preventable visits & 0.08 & Number of outpatient visits & 0.09 \\
\hline Number of primary care treatable visits & 0.08 & Number of overall visits & 0.08 \\
\hline Female & 0.04 & Number of non-emergent visits & 0.08 \\
\hline Number of non-emergent visits & 0.04 & Number of emergent, preventable visits & 0.06 \\
\hline Week of lottery sign up & 0.04 & Number of off-hours visits & 0.06 \\
\hline Pre-lottery SNAP recipient & 0.02 & Pre-lottery SNAP benefit amount (\$) & 0.06 \\
\hline Number of emergent, preventable visits & 0.02 & Age (years) & 0.03 \\
\hline Number of visits (chronic conditions) & 0.01 & Number of inpatient visits & 0.03 \\
\hline Number of inpatient visits & 0.01 & Number of on-hours visits & 0.03 \\
\hline Number of outpatient visits & 0.01 & Number of visits (chronic conditions) & 0.02 \\
\hline Number of off-hours visits & 0.01 & Number of visits (injury) & 0.02 \\
\hline Number of overall visits & 0.01 & Week of lottery sign up & 0.01 \\
\hline Number of on-hours visits & 0.01 & Gave phone number & 0.01 \\
\hline Gave phone number & 0.01 & Female & 0.01 \\
\hline Signed up self for lottery & 0.01 & Number of visits (abdominal pain) & 0.00 \\
\hline Number of visits (injury) & 0.01 & Number of visits (psychiatric conditions) & 0.00 \\
\hline Pre-lottery TANF benefit amount (\$) & 0.00 & Pre-lottery SNAP recipient & 0.00 \\
\hline English as preferred language & 0.00 & Number of visits (chest pain) & 0.00 \\
\hline Number of visits (mood disorders) & 0.00 & Pre-lottery TANF benefit amount (\$) & 0.00 \\
\hline Number of visits (psychiatric conditions) & 0.00 & Number of visits (skin conditions) & 0.00 \\
\hline Number ambulatory-care-sensitive visits & 0.00 & Number of visits (back pain) & 0.00 \\
\hline Number of visits (abdominal pain) & 0.00 & Number of visits (headache) & 0.00 \\
\hline Number of visits (skin conditions) & 0.00 & Number of visits (mood disorders) & 0.00 \\
\hline Pre-lottery TANF recipient & 0.00 & Number ambulatory-care-sensitive visits & 0.00 \\
\hline Number of visits (chest pain) & 0.00 & Signed up self for lottery & 0.00 \\
\hline Number of visits (back pain) & 0.00 & Pre-lottery TANF recipient & 0.00 \\
\hline Number of visits (headache) & 0.00 & English as preferred language & 0.00 \\
\hline Provided P.O. box address & 0.00 & Provided P.O. box address & 0.00 \\
\hline
\end{tabular}

Notes: This table shows the top variable importance scores of all characteristics for growing the generalized random forests used to estimate the ITE of winning the lottery for overall ED visits. The variable importance measure is a simple weighted sum of the proportion of times a variable is used in a splitting step at each depth in growing the causal forest, thus, capturing how important a variable is for driving treatment effect heterogeneity. The sample consists of 24,615 individuals in the Taubman et al. (2014) sample with non-missing information on pre-lottery emergency department utilization and SNAP/TANF receipt. 